\title{
EXTREMAL BEHAVIOUR OF CHAOTIC DYNAMICS
}

\author{
JORGE MILHAZES FREITAS
}

\begin{abstract}
We present a review of recent results regarding the existence of Extreme Value Laws for stochastic processes arising from dynamical systems. We gather all the conditions on the dependence structure of stationary stochastic processes in order to obtain both the distributional limit for partial maxima and the convergence of point processes of rare events. We also discuss the existence of clustering which can be detected by an Extremal Index less than 1 and relate it with the occurrence of rare events around periodic points. We also present the connection between the existence of Extreme Value Laws for certain dynamically defined stationary stochastic processes and the existence of Hitting Times Statistics (or Return Times Statistics). Finally, we make a complete description of the extremal behaviour of expanding and piecewise expanding systems by giving a dichotomy regarding the types of Extreme Value Laws that apply. Namely, we show that around periodic points we have an Extremal Index less than 1 and at very other point we have an Extremal Index equal to 1.
\end{abstract}

\section{INTRODUCTION}

The purpose of this work is to compile a series of recent results regarding the theory of extreme events for dynamical systems. Extreme means that we are interested in the occurrence of rare but possibly catastrophic events. This motivates its applications to situations where risk assessment is rather crucial.

Instead of focusing on means and average behaviour of the system, often ruled by central limit theorems, in this approach, we are interested on the largest (or smallest) observations of sample data in order to understand the extremal behaviour of the system by obtaining corresponding distributional limits.

The starting point of our analysis is a stationary stochastic process arising from a chaotic dynamical system simply by evaluating an observable function along the orbits of the

Date: March 1, 2013.

2000 Mathematics Subject Classification. 37A25, 37A50, 37C40, 37B20, 60G70, 60 G10.

Key words and phrases. Extreme Value Laws, Hitting/Return Time Statistics, Extremal Index, Point Processes, Expanding and Piecewise Expanding Dynamical Systems, Dichotomy.

The author was partially supported by FCT (Portugal) grant SFRH/BPD/66040/2009, by FCT projects PTDC/MAT/099493/2008 and PTDC/MAT/120346/2010, which are financed by national and European Community structural funds through the programs FEDER and COMPETE . The author also acknowledges the support of CMUP, which is financed by FCT (Portugal) through the programs POCTI and POSI, with national and European Community structural funds. The author wishes to thank Mike Todd for his comments and suggestions. 
system, as explained in Section 3 , below. Then the extremal behaviour is analysed by studying the distributional limit of the partial maximum of such stochastic processes or by investigating the limit of point processes counting the number of exceedances of high thresholds during some time interval.

In the context of dynamical systems, the study of Extreme Value Laws (EVL) is a quite recent topic. It appeared first in the pioneering paper of Collet, [16], which has been an inspiration for plenty of our research on this issue. Then the subject was further addressed and developed in $25,26,54,27,28,33,38,30,29,39,21,22,20,49,41,8,19$. The main results stated here are spread over the papers [26, 27, 28, 30, 29, 8]. We highlight that, in [27, a full connection was established between the existence of EVL and the existence of Hitting Time Statistics (HTS), which has to do with distributional limits for the elapsed time before the orbits of the system hit some shrinking targets in the phase space. We remark that while the connection between these two limit laws had already been noted by Collet in [16], the complete equivalence was formally established and further developed in [27, 28]. This alternative approach to the study of occurrence of rare events is tied with recurrence and the existence of Return Times Statistics (RTS), which has received plenty of attention from dynamicists since the early 1990s.

The structure of this paper is as follows. In Section 2, we introduce the basic definitions and classical results regarding the theory of EVL for general stochastic processes. In Section 3, we give conditions in order to obtain EVL both in the absence and presence of clustering; we define the rare events point process and also give conditions for its convergence in distribution to a standard Poisson process (no clustering) or to a compound Poisson distribution (with clustering); we introduce a new notation and prove some abstract lemmata that will allow us to make a common proof of the existence of EVL both in the presence and absence of clustering (Section 3.4). In Section 4, we give the link between EVL and HTS for general equilibrium states as in [28]. In Section 5, we start by making a a brief literature review where we discuss the systems to which we can apply the theory and prove the existence of laws of rare events. Then, we consider the case of expanding and piecewise expanding maps for which we can actually prove a dichotomy regarding the type of limiting law we get, depending on whether the action develops around periodic or non periodic points. Moreover, in Section 5.1 we show thoroughly how to check the several mixing conditions introduced in Section 3 from sufficiently fast decay of correlations.

\section{Extreme VAlues - DEFinitions AND CONCEPTS}

Let $X_{0}, X_{1}, \ldots$ be a stationary stochastic process. We denote by $F$ the cumulative distribution function (d.f.) of $X_{0}$, i.e., $F(x)=\mathbb{P}\left(X_{0} \leq x\right)$. Given any d.f. $F$, let $\bar{F}=1-F$ and $u_{F}$ denote the right endpoint of the d.f. $F$, i.e., $u_{F}=\sup \{x: F(x)<1\}$. We say we have an exceedance of the threshold $u<u_{F}$ whenever

$$
U(u):=\left\{X_{0}>u\right\}
$$

occurs. 
We define a new sequence of random variables $M_{1}, M_{2}, \ldots$ given by

$$
M_{n}=\max \left\{X_{0}, \ldots, X_{n-1}\right\} .
$$

Definition 1. We say that we have an Extreme Value Law (EVL) for $M_{n}$ if there is a non-degenerate d.f. $H: \mathbb{R} \rightarrow[0,1]$ with $H(0)=0$ and, for every $\tau>0$, there exists a sequence of levels $u_{n}=u_{n}(\tau), n=1,2, \ldots$, such that

$$
n \mathbb{P}\left(X_{0}>u_{n}\right) \rightarrow \tau, \text { as } n \rightarrow \infty,
$$

and for which the following holds:

$$
\mathbb{P}\left(M_{n} \leq u_{n}\right) \rightarrow \bar{H}(\tau), \text { as } n \rightarrow \infty .
$$

where the convergence is meant at the continuity points of $H(\tau)$.

The motivation for using a normalising sequence $\left(u_{n}\right)_{n \in \mathbb{N}}$ satisfying $(2.3)$ comes from the case when $X_{0}, X_{1}, \ldots$ are independent and identically distributed (i.i.d.).

2.1. The i.i.d. setting and the classical results. In the i.i.d. setting, it is clear that $\mathbb{P}\left(M_{n} \leq u\right)=(F(u))^{n}$, where $F$ is the d.f. of $X_{0}$, i.e., $F(x):=\mathbb{P}\left(X_{0} \leq x\right)$. Hence, condition (2.3) implies that

$$
\mathbb{P}\left(M_{n} \leq u_{n}\right)=\left(1-\mathbb{P}\left(X_{0}>u_{n}\right)\right)^{n} \sim\left(1-\frac{\tau}{n}\right)^{n} \rightarrow \mathrm{e}^{-\tau},
$$

as $n \rightarrow \infty$. Moreover, the reciprocal is also true (see [46, Theorem 1.5.1] for more details). Note that in this case $H(\tau)=1-\mathrm{e}^{-\tau}$ is the standard exponential d.f.

Moreover, the sequences of real numbers $u_{n}=u_{n}(\tau), n=1,2, \ldots$, are usually taken to be one parameter linear families like

$$
u_{n}=y / a_{n}+b_{n}
$$

where $y \in \mathbb{R}$ and $a_{n}>0$, for all $n \in \mathbb{N}$. In fact, in the classical theory, one considers the convergence of probabilities of the form

$$
\mathbb{P}\left(a_{n}\left(M_{n}-b_{n}\right) \leq y\right) .
$$

In this case, the Extremal Types Theorem ([24, 32]) says that, whenever the variables $X_{i}$ are i.i.d, if for some constants $a_{n}>0, b_{n}$, we have

$$
\mathbb{P}\left(a_{n}\left(M_{n}-b_{n}\right) \leq y\right) \rightarrow G(y),
$$

where the convergence occurs at continuity points of $G$, and $G$ is non degenerate (meaning that there is no $y_{0} \in \mathbb{R}$ such that $G\left(y_{0}\right)=1$ and $G(y)=0$, for all $\left.y<y_{0}\right)$, then $G(y)=$ $\mathrm{e}^{-\tau(y)}$, where $\tau(y)$ is of one of the following three types (for some $\alpha>0$ ):

$$
\tau_{1}(y)=\mathrm{e}^{-y} \text { for } y \in \mathbb{R}, \quad \tau_{2}(y)=y^{-\alpha} \text { for } y>0 \quad \text { and } \quad \tau_{3}(y)=(-y)^{\alpha} \text { for } y \leq 0 .
$$

We emphasise that as observed in [32], for i.i.d. sequences of random variables, the limiting distribution type of the partial maxima is completely determined by the tail of the d.f. $F$. Namely, as can also be found in [46, Theorem 1.6.2], in order to obtain the respective 
domain of attraction for maxima we have the following sufficient and necessary conditions on the tail of the d.f. $F$ :

Type $1 G(y)=\mathrm{e}^{-\tau_{1}(y)}$ (Gumbel) iff there exists some strictly positive function $h: \mathbb{R} \rightarrow \mathbb{R}$ such that for all $y \in \mathbb{R}$

$$
\lim _{s \rightarrow u_{F}} \frac{\bar{F}(s+y h(s))}{\bar{F}(s)}=\mathrm{e}^{-y}
$$

Type $2 G(y)=\mathrm{e}^{-\tau_{2}(y)}$ (Fréchet) iff $u_{F}=+\infty$ and there exists $\beta>0$ such that for all $y>0$

$$
\lim _{s \rightarrow u_{f}} \frac{\bar{F}(s y)}{\bar{F}(s)}=y^{-\beta}
$$

Type $3 G(y)=\mathrm{e}^{-\tau_{3}(y)}$ (Weibull) iff $u_{F}<+\infty$ and there exists $\beta>0$ such that for all $y>0$

$$
\lim _{s \rightarrow 0} \frac{\bar{F}\left(u_{F}-s y\right)}{\bar{F}\left(u_{F}-s\right)}=y^{\beta}
$$

Moreover, in [46, Corollary 1.6.3] one can find specific formulas for the normalising constants $a_{n}$ and $b_{n}$ so that the respective extreme limit laws apply.

2.2. Stationary sequences and dependence conditions. When $X_{0}, X_{1}, X_{2}, \ldots$ are not independent but satisfy some mixing condition $D\left(u_{n}\right)$ introduced by Leadbetter in [44] then something can still be said about $H$. Let $F_{i_{1}, \ldots, i_{n}}$ denote the joint d.f. of $X_{i_{1}}, \ldots, X_{i_{n}}$, and set $F_{i_{1}, \ldots, i_{n}}(u)=F_{i_{1}, \ldots, i_{n}}(u, \ldots, u)$.

Condition $\left(D\left(u_{n}\right)\right)$. We say that $D\left(u_{n}\right)$ holds for the sequence $X_{0}, X_{1}, \ldots$ if for any integers $i_{1}<\ldots<i_{p}$ and $j_{1}<\ldots<j_{k}$ for which $j_{1}-i_{p}>m$, and any large $n \in \mathbb{N}$,

$$
\left|F_{i_{1}, \ldots, i_{p}, j_{1}, \ldots, j_{k}}\left(u_{n}\right)-F_{i_{1}, \ldots, i_{p}}\left(u_{n}\right) F_{j_{1}, \ldots, j_{k}}\left(u_{n}\right)\right| \leq \gamma(n, t)
$$

where $\gamma\left(n, t_{n}\right) \underset{n \rightarrow \infty}{\longrightarrow} 0$, for some sequence $t_{n}=o(n)$.

If $D\left(u_{n}\right)$ holds for $X_{0}, X_{1}, \ldots$ and the limit 2.4 exists for some $\tau>0$ then there exists $0 \leq \theta \leq 1$ such that $\bar{H}(\tau)=\mathrm{e}^{-\theta \tau}$ for all $\tau>0$ (see [45, Theorem 2.2] or [46, Theorem 3.7.1]).

Definition 2. We say that $X_{0}, X_{1}, \ldots$ has an Extremal Index (EI) $0 \leq \theta \leq 1$ if we have an EVL for $M_{n}$ with $\bar{H}(\tau)=\mathrm{e}^{-\theta \tau}$ for all $\tau>0$.

The notion of the EI was latent in the work of Loynes [48] but was established formally by Leadbetter in [45]. It gives a measure of the strength of the dependence of $X_{0}, X_{1}, \ldots$, so that $\theta=1$ indicates that the process has practically no memory while $\theta=0$, conversely, reveals extremely long memory. Another way of looking at the EI is that it gives some indication of how much exceedances of high levels have a tendency to "cluster". Namely, for $\theta>0$ this interpretation of the EI is that $\theta^{-1}$ is the mean number of exceedances of a high level in a cluster of large observations, i.e., is the "mean size of the clusters". 
In fact, as shown in [44], if $D\left(u_{n}\right)$ holds and in addition an anti clustering condition $D^{\prime}\left(u_{n}\right)$ (see definition below) also holds, one can show that the EI is 1 . Let $\left(k_{n}\right)_{n \in \mathbb{N}}$ be a sequence of integers such that

$$
k_{n} \rightarrow \infty \text { and } k_{n} t_{n}=o(n) .
$$

Condition $\left(D^{\prime}\left(u_{n}\right)\right)$. We say that $D^{\prime}\left(u_{n}\right)$ holds for the sequence $X_{0}, X_{1}, X_{2}, \ldots$ if there exists a sequence $\left\{k_{n}\right\}_{n \in \mathbb{N}}$ satisfying 2.12 and such that

$$
\lim _{n \rightarrow \infty} n \sum_{j=1}^{\left\lfloor n / k_{n}\right\rfloor} \mathbb{P}\left(X_{0}>u_{n}, X_{j}>u_{n}\right)=0 .
$$

Hence, under conditions $D\left(u_{n}\right)$ and $D^{\prime}\left(u_{n}\right)$ we have an EVL for $M_{n}$ such that $\bar{H}(\tau)=\mathrm{e}^{-\tau}$, as in the independent case.

Condition $D\left(u_{n}\right)$ can be seen as a long range mixing condition, requiring that two events corresponding to no exceedances among two groups of random variables, separated by a time gap, become more and more independent as the size of the gap increases. On the other hand, condition $D^{\prime}\left(u_{n}\right)$ is a short range dependence condition in the sense that the occurrence of an exceedance of high thresholds should not be "dependent" of the occurrence of another nearby exceedance, in the time line. In other words, if we break the first $n$ random variables into blocks of size $\left\lfloor n / k_{n}\right\rfloor$, then $D^{\prime}\left(u_{n}\right)$ restricts the existence of more than one exceedance in each block.

\section{EXtreme VAlues For DyNAMiCALly DEFined STOCHAStiC PROCESSES}

Take a system $(\mathcal{X}, \mathcal{B}, \mathbb{P}, f)$, where $\mathcal{X}$ is a Riemannian manifold, $\mathcal{B}$ is the Borel $\sigma$-algebra, $f: \mathcal{X} \rightarrow \mathcal{X}$ is a measurable map and $\mathbb{P}$ an $f$-invariant probability measure.

Suppose that the time series $X_{0}, X_{1}, \ldots$ arises from such a system simply by evaluating a given observable $\varphi: \mathcal{X} \rightarrow \mathbb{R} \cup\{ \pm \infty\}$ along the orbits of the system, or in other words, the time evolution given by successive iterations by $f$ :

$$
X_{n}=\varphi \circ f^{n}, \quad \text { for each } n \in \mathbb{N} .
$$

Clearly, $X_{0}, X_{1}, \ldots$ defined in this way is not an independent sequence. However, $f$ invariance of $\mathbb{P}$ guarantees that this stochastic process is stationary.

We assume that the r.v. $\varphi: \mathcal{X} \rightarrow \mathbb{R} \cup\{ \pm \infty\}$ achieves a global maximum at $\zeta \in \mathcal{X}$ (we allow $\varphi(\zeta)=+\infty)$. We also assume that $\varphi$ and $\mathbb{P}$ are sufficiently regular so that:

(R1) for $u$ sufficiently close to $u_{F}:=\varphi(\zeta)$, the event

$$
U(u)=\left\{X_{0}>u\right\}=\{x \in \mathcal{X}: \varphi(x)>u\}
$$

corresponds to a topological ball centred at $\zeta$. Moreover, the quantity $\mathbb{P}(U(u))$, as a function of $u$, varies continuously on a neighbourhood of $u_{F}$. 
(R2) If $\zeta \in \mathcal{X}$ is a repelling periodic point, of prime period ${ }^{1} p \in \mathbb{N}$, then we have that the periodicity of $\zeta$ implies that for all large $u,\left\{X_{0}>u\right\} \cap f^{-p}\left(\left\{X_{0}>u\right\}\right) \neq \emptyset$ and the fact that the prime period is $p$ implies that $\left\{X_{0}>u\right\} \cap f^{-j}\left(\left\{X_{0}>u\right\}\right)=\emptyset$ for all $j=1, \ldots, p-1$. Moreover, the fact that $\zeta$ is repelling means that we have backward contraction implying that there exists $0<\theta<1$ so that $\bigcap_{j=0}^{i} f^{-j p}\left(X_{0}>u\right)$ is another ball of smaller radius around $\zeta$ with

$$
\mathbb{P}\left(\bigcap_{j=0}^{i} f^{-j p}\left(X_{0}>u\right)\right) \sim(1-\theta)^{i} \mathbb{P}\left(X_{0}>u\right),
$$

for all $u$ sufficiently large.

We are interested in studying the extremal behaviour of the stochastic process $X_{0}, X_{1}, \ldots$ which is tied with the occurrence of exceedances of high levels $u$. The occurrence of an exceedance at time $j \in \mathbb{N}_{0}$ means that the event $\left\{X_{j}>u\right\}$ occurs, where $u$ is close to $u_{F}$. Observe that a realisation of the stochastic process $X_{0}, X_{1}, \ldots$ is achieved if we pick, at random and according to the measure $\mathbb{P}$, a point $x \in \mathcal{X}$, compute its orbit and evaluate $\varphi$ along it. Then saying that an exceedance occurs at time $j$ means that the orbit of the point $x$ hits the ball $U(u)$ at time $j$, i.e., $f^{j}(x) \in U(u)$.

3.1. EVLs for dynamical stochastic processes in the absence of clustering. The first natural approach to study the extremal properties of stochastic processes defined as in (3.1), would be to apply the dependence conditions of Leadbetter for general stationary sequences described in Section 2.2. However, since the rates of mixing for dynamical systems are usually given by decay of correlations of observables in certain given classes of functions, it turns out that condition $D\left(u_{n}\right)$ is too strong to be checked for chaotic systems whose mixing rates are known only through decay of correlations (see Section 5.1 for more details). For that reason, motivated by Collet's work [16], in [26] the authors suggested a condition $D_{2}\left(u_{n}\right)$ which together with $D^{\prime}\left(u_{n}\right)$ was enough to prove the existence of an exponential EVL $\left(\bar{H}(\tau)=\mathrm{e}^{-\tau}\right)$ for maxima around non-periodic points $\zeta$.

Condition $\left(D_{2}\left(u_{n}\right)\right)$. We say that $D_{2}\left(u_{n}\right)$ holds for the sequence $X_{0}, X_{1}, \ldots$ if for all $\ell, t$ and $n$

$$
\left|\mathbb{P}\left(X_{0}>u_{n} \cap \max \left\{X_{t}, \ldots, X_{t+\ell-1} \leq u_{n}\right\}\right)-\mathbb{P}\left(X_{0}>u_{n}\right) \mathbb{P}\left(M_{\ell} \leq u_{n}\right)\right| \leq \gamma(n, t),
$$

where $\gamma(n, t)$ is decreasing in $t$ for each $n$ and $n \gamma\left(n, t_{n}\right) \rightarrow 0$ when $n \rightarrow \infty$ for some sequence $t_{n}=o(n)$.

Condition $D_{2}\left(u_{n}\right)$ is much weaker than the original $D\left(u_{n}\right)$, and it is easy to show that it follows easily from sufficiently fast decay of correlations (see Section 5.1).

Theorem 1 ( [26, Theorem 1]). If conditions $D_{2}\left(u_{n}\right)$ and $D^{\prime}\left(u_{n}\right)$ hold for $X_{0}, X_{1}, \ldots$ then there exists an EVL for $M_{n}$ and $H(\tau)=1-e^{-\tau}$.

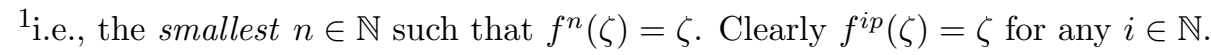


Observe that while $D\left(u_{n}\right)$ imposes some rate for the dependence of two blocks of r.v. separated by a time gap which is independent of the size of the blocks, condition $D_{2}\left(u_{n}\right)$ requires something similar but only when the first block is reduced to one r.v. only. This detail turns out to be crucial when proving $D_{2}\left(u_{n}\right)$ from decay of correlations as can be seen in Section 5.1. The interesting fact is that we can replace $D\left(u_{n}\right)$ by $D_{2}\left(u_{n}\right)$ in 45 , Theorem 1.2] and the conclusion still holds. The idea is that condition $D^{\prime}\left(u_{n}\right)$, instead of being used once as in the original proof of Leadbetter, it is used twice. In one of the instances it is used in conjunction with $D_{2}\left(u_{n}\right)$ to produce the same effect as $D\left(u_{n}\right)$ alone.

3.2. Clustering and periodicity. In the results mentioned above, condition $D^{\prime}\left(u_{n}\right)$ prevented the existence of clusters of exceedances, which implies for example that the EVL was a standard exponential $\bar{H}(\tau)=\mathrm{e}^{-\tau}$. However, when $D^{\prime}\left(u_{n}\right)$ does not hold, clustering of exceedances is responsible for the appearance of a parameter $0<\theta<1$ in the EVL which now is written as $\bar{H}(\tau)=\mathrm{e}^{-\theta \tau}$.

In [30], the authors established a connection between the existence of an EI less than 1 and periodic behaviour. This was later generalised for rare events point processes in [29]. The main obstacle when dealing with periodic points is that they create plenty of dependence in the short range. In particular, using (R2) we have that for all $u$ sufficiently large

$$
\mathbb{P}\left(\left\{X_{0}>u\right\} \cap\left\{X_{p}>u\right\}\right) \sim(1-\theta) \mathbb{P}\left(X_{0}>u\right) .
$$

which implies that $D^{\prime}\left(u_{n}\right)$ is not satisfied, since for the levels $u_{n}$ as in (2.3) it follows that

$$
n \sum_{j=1}^{\left[n / k_{n}\right]} \mathbb{P}\left(X_{0}>u_{n}, X_{j}>u_{n}\right) \geq n \mathbb{P}\left(X_{0}>u_{n}, X_{p}>u_{n}\right) \underset{n \rightarrow \infty}{\longrightarrow}(1-\theta) \tau .
$$

Recalling the discussion at the end of Section 3.1, condition $D^{\prime}\left(u_{n}\right)$ was essential to allow the replacement of $D\left(u_{n}\right)$ by $D_{2}\left(u_{n}\right)$ in order to use decay of correlations to get the result. To overcome this difficulty around periodic points we make a key observation that roughly speaking tells us that around periodic points one just needs to replace the topological ball $\left\{X_{0}>u_{n}\right\}$ by the topological annulus

$$
Q_{p}(u):=\left\{X_{0}>u, X_{p} \leq u\right\} .
$$

Then much of the analysis works out as in the absence of clustering. Note that $Q_{p}(u)$ is obtained by removing from $U(u)$ the points that were doomed to return after $p$ steps, which form the smaller ball $U(u) \cap f^{-p}(U(u))$. Then, the crucial observation is that the limit law corresponding to no entrances up to time $n$ into the ball $U\left(u_{n}\right)$ is equal to the limit law corresponding to no entrances into the annulus $Q_{p}\left(u_{n}\right)$ up to time $n$.

In what follows for every $A \in \mathcal{B}$, we denote the complement of $A$ as $A^{c}:=\mathcal{X} \backslash A$. For $s \leq \ell \in \mathbb{N}_{0}$, we define

$$
\mathscr{Q}_{p, s, \ell}(u)=\bigcap_{i=s}^{s+\ell-1} f^{-i}\left(Q_{p}(u)\right)^{c}
$$


which corresponds to no entrances in the annulus from time $s$ to $s+\ell-1$. Sometimes to abbreviate we also write: $\mathscr{Q}_{\ell}(u):=\mathscr{Q}_{p, 0, \ell}(u)$.

Theorem 2 ([30, Proposition 1]). Let $X_{0}, X_{1}, \ldots$ be a stochastic process defined by (3.1) where $\varphi$ achieves a global maximum at a repelling periodic point $\zeta \in \mathcal{X}$, of prime period $p \in \mathbb{N}$, so that conditions (R1) and (R2) above hold. Let $\left(u_{n}\right)_{n}$ be a sequence of levels such that (2.3) holds. Then,

$$
\lim _{n \rightarrow \infty} \mathbb{P}\left(M_{n} \leq u_{n}\right)=\lim _{n \rightarrow \infty} \mathbb{P}\left(\mathscr{Q}_{n}\left(u_{n}\right)\right)
$$

Hence, the idea to cope with clustering caused by periodic points is to adapt conditions $D_{2}\left(u_{n}\right)$ and $D^{\prime}\left(u_{n}\right)$, letting annuli replace balls. In order to make the theory as general as possible, motivated by the above considerations for stochastic processes generated by dynamical systems around periodic points, some abstract conditions were given in [30] to prove the existence of an EI less than 1 for general stationary stochastic processes.

The first one establishes exactly the type of periodic behaviour assumed, namely:

Condition $\left(\mathrm{SP}_{p, \theta}\left(u_{n}\right)\right)$. We say that $X_{0}, X_{1}, X_{2}, \ldots$ satisfies condition $\operatorname{SP}_{p, \theta}\left(u_{n}\right)$ for $p \in \mathbb{N}$ and $\theta \in[0,1]$ if

$$
\lim _{n \rightarrow \infty} \sup _{1 \leq j<p} \mathbb{P}\left(X_{j}>u_{n} \mid X_{0}>u_{n}\right)=0 \quad \text { and } \quad \lim _{n \rightarrow \infty} \mathbb{P}\left(X_{p}>u_{n} \mid X_{0}>u_{n}\right) \rightarrow(1-\theta)
$$

and moreover

$$
\lim _{n \rightarrow \infty} \sum_{i=0}^{\left[\frac{n-1}{p}\right]} \mathbb{P}\left(X_{0}>u_{n}, X_{p}>u_{n}, X_{2 p}>u_{n}, \ldots, X_{i p}>u_{n}\right)=0 .
$$

Condition (3.5), when $\theta<1$, imposes some sort of periodicity of period $p$ among the exceedances of high levels $u_{n}$, since if at some point the process exceeds the high level $u_{n}$, then, regardless of how high $u_{n}$ is, there is always a strictly positive probability of another exceedance occurring at the (finite) time $p$. In fact, if the process is generated by a deterministic dynamical system $f: \mathcal{X} \rightarrow \mathcal{X}$ and $f$ is continuous then (3.5) implies that $\zeta$ is a periodic point of period $p$, i.e., $f^{p}(\zeta)=\zeta$.

The next two conditions concern to the dependence structure of $X_{0}, X_{1}, \ldots$ and can be described as being obtained from $D_{2}\left(u_{n}\right)$ and $D^{\prime}\left(u_{n}\right)$ by replacing balls by annuli.

Condition $\left(D^{p}\left(u_{n}\right)\right)$. We say that $D^{p}\left(u_{n}\right)$ holds for the sequence $X_{0}, X_{1}, X_{2}, \ldots$ if for any integers $\ell, t$ and $n$

$$
\left|\mathbb{P}\left(Q_{p, 0}\left(u_{n}\right) \cap \mathscr{Q}_{p, t, \ell}\left(u_{n}\right)\right)-\mathbb{P}\left(Q_{p, 0}\left(u_{n}\right)\right) \mathbb{P}\left(\mathscr{Q}_{p, 0, \ell}\left(u_{n}\right)\right)\right| \leq \gamma(n, t),
$$

where $\gamma(n, t)$ is non increasing in $t$ for each $n$ and $n \gamma\left(n, t_{n}\right) \rightarrow 0$ as $n \rightarrow \infty$ for some sequence $t_{n}=o(n)$.

As with $D_{2}\left(u_{n}\right)$, the main advantage of this condition when compared to Leadbetter's $D\left(u_{n}\right)$ (or others of the same sort) is that it follows directly from sufficiently fast decay of correlations as observed in Section 5.1, on the contrary to $D\left(u_{n}\right)$. 
Assuming $D^{p}\left(u_{n}\right)$ holds let $\left(k_{n}\right)_{n \in \mathbb{N}}$ be a sequence of integers such that 2.12 holds.

Condition $\left(D_{p}^{\prime}\left(u_{n}\right)\right)$. We say that $D_{p}^{\prime}\left(u_{n}\right)$ holds for the sequence $X_{0}, X_{1}, X_{2}, \ldots$ if there exists a sequence $\left\{k_{n}\right\}_{n \in \mathbb{N}}$ satisfying $(2.12$ and such that

$$
\lim _{n \rightarrow \infty} n \sum_{j=1}^{\left[n / k_{n}\right]} \mathbb{P}\left(Q_{p, 0}\left(u_{n}\right) \cap Q_{p, j}\left(u_{n}\right)\right)=0 .
$$

One of the main results in [30] is:

Theorem 3 ([30, Theorem 1]). Let $\left(u_{n}\right)_{n \in \mathbb{N}}$ be such that $(2.3)$ holds. Consider a stationary stochastic process $X_{0}, X_{1}, \ldots$ satisfying $S P_{p, \theta}\left(u_{n}\right)$ for some $p \in \mathbb{N}$, and $\theta \in(0,1)$. Assume further that conditions $D^{p}\left(u_{n}\right)$ and $D_{p}^{\prime}\left(u_{n}\right)$ hold. Then

$$
\lim _{n \rightarrow \infty} \mathbb{P}\left(M_{n} \leq u_{n}\right)=\lim _{n \rightarrow \infty} \mathbb{P}\left(\mathscr{Q}_{p, 0, n}\left(u_{n}\right)\right)=\mathrm{e}^{-\theta \tau} .
$$

3.3. Point processes of rare events. If we enrich the process by considering multiple exceedances we are lead to point processes of rare events counting the number of exceedances in a certain time frame. For every $A \subset \mathbb{R}$ we define

$$
\mathscr{N}_{u}(A):=\sum_{i \in A \cap \mathbb{N}_{0}} \mathbf{1}_{X_{i}>u}
$$

In the particular case where $A=I=[a, b)$ we simply write $\mathscr{N}_{u, a}^{b}:=\mathscr{N}_{u}([a, b))$. Observe that $\mathscr{N}_{u, 0}^{n}$ counts the number of exceedances amongst the first $n$ observations of the process $X_{0}, X_{1}, \ldots, X_{n}$ or, in other words, the number of entrances in $U(u)$ up to time $n$. Also, note that

$$
\left\{\mathscr{N}_{u, 0}^{n}=0\right\}=\left\{M_{n} \leq u\right\}=\left\{r_{U(u)}>n\right\}
$$

In order to define a point process that through (3.9) captures the essence of an EVL and HTS, we need to re-scale time using the factor $v:=1 / \mathbb{P}(X>u)$ given by Kac's Theorem. However, before we give the definition, we need some formalism. Let $\mathcal{S}$ denote the semiring of subsets of $\mathbb{R}_{0}^{+}$whose elements are intervals of the type $[a, b)$, for $a, b \in \mathbb{R}_{0}^{+}$. Let $\mathcal{R}$ denote the ring generated by $\mathcal{S}$. Recall that for every $J \in \mathcal{R}$ there are $k \in \mathbb{N}$ and $k$ intervals $I_{1}, \ldots, I_{k} \in \mathcal{S}$ such that $J=\cup_{i=1}^{k} I_{j}$. In order to fix notation, let $a_{j}, b_{j} \in \mathbb{R}_{0}^{+}$ be such that $I_{j}=\left[a_{j}, b_{j}\right) \in \mathcal{S}$. For $I=[a, b) \in \mathcal{S}$ and $\alpha \in \mathbb{R}$, we denote $\alpha I:=[\alpha a, \alpha b)$ and $I+\alpha:=[a+\alpha, b+\alpha)$. Similarly, for $J \in \mathcal{R}$ define $\alpha J:=\alpha I_{1} \cup \cdots \cup \alpha I_{k}$ and $J+\alpha:=\left(I_{1}+\alpha\right) \cup \cdots \cup\left(I_{k}+\alpha\right)$.

Definition 3. We define the rare event point process (REPP) by counting the number of exceedances (or hits to $U\left(u_{n}\right)$ ) during the (re-scaled) time period $v_{n} J \in \mathcal{R}$, where $J \in \mathcal{R}$. To be more precise, for every $J \in \mathcal{R}$, set

$$
N_{n}(J):=\mathscr{N}_{u_{n}}\left(v_{n} J\right)=\sum_{j \in v_{n} J \cap \mathbb{N}_{0}} \mathbf{1}_{X_{j}>u_{n}} .
$$


Under similar dependence conditions to the ones just seen above, the REPP just defined converges in distribution to a standard Poisson process, when no clustering is involved and to a compound Poisson process with intensity $\theta$ and a geometric multiplicity d.f., otherwise. For completeness, we define here what we mean by a Poisson and a compound Poisson process. (See [40] for more details).

Definition 4. Let $T_{1}, T_{2}, \ldots$ be an i.i.d. sequence of random variables with common exponential distribution of mean $1 / \theta$. Let $D_{1}, D_{2}, \ldots$ be another i.i.d. sequence of random variables, independent of the previous one, and with d.f. $\pi$. Given these sequences, for $J \in \mathcal{R}$, set

$$
N(J)=\int \mathbf{1}_{J} d\left(\sum_{i=1}^{\infty} D_{i} \delta_{T_{1}+\ldots+T_{i}}\right),
$$

where $\delta_{t}$ denotes the Dirac measure at $t>0$. Whenever we are in this setting, we say that $N$ is a compound Poisson process of intensity $\theta$ and multiplicity d.f. $\pi$.

Remark 1. In this paper, the multiplicity will always be integer valued which means that $\pi$ is completely defined by the values $\pi_{k}=\mathbb{P}\left(D_{1}=k\right)$, for every $k \in \mathbb{N}_{0}$. Note that, if $\pi_{1}=1$ and $\theta=1$, then $N$ is the standard Poisson process and, for every $t>0$, the random variable $N([0, t))$ has a Poisson distribution of mean $t$.

Remark 2. When clustering is involved, we will see that $\pi$ is actually a geometric distribution of parameter $\theta \in(0,1]$, i.e., $\pi_{k}=\theta(1-\theta)^{k}$, for every $k \in \mathbb{N}_{0}$. This means that, as in [35], here, the random variable $N([0, t))$ follows a Pólya-Aeppli distribution, i.e.:

$$
\mathbb{P}(N([0, t))=k)=\mathrm{e}^{-\theta t} \sum_{j=1}^{k} \theta^{j}(1-\theta)^{k-j} \frac{(\theta t)^{j}}{j !}\left(\begin{array}{c}
k-1 \\
j-1
\end{array}\right),
$$

for all $k \in \mathbb{N}$ and $\mathbb{P}(N([0, t))=0)=\mathrm{e}^{-\theta t}$.

3.3.1. The absence of clustering. When $D^{\prime}\left(u_{n}\right)$ holds, since there is no clustering, then, due to a criterion proposed by Kallenberg [40, Theorem 4.7], which applies only to simple point processes, without multiple events, we can simply adjust condition $D_{2}\left(u_{n}\right)$ to this scenario of multiple exceedances in order to prove that the REPP converges in distribution to a standard Poisson process. We denote this adapted condition by:

Condition $\left(D_{3}\left(u_{n}\right)\right)$. Let $A \in \mathcal{R}$ and $t \in \mathbb{N}$. We say that $D_{3}\left(u_{n}\right)$ holds for the sequence $X_{0}, X_{1}, \ldots$ if

$$
\left|\mathbb{P}\left(\left\{X_{0}>u_{n}\right\} \cap\{\mathscr{N}(A+t)=0\}\right)-\mathbb{P}\left(\left\{X_{0}>u_{n}\right\}\right) \mathbb{P}(\mathscr{N}(A)=0)\right| \leq \gamma(n, t),
$$

where $\gamma(n, t)$ is nonincreasing in $t$ for each $n$ and $n \gamma\left(n, t_{n}\right) \rightarrow 0$ as $n \rightarrow \infty$ for some sequence $t_{n}=o(n)$, which means that $t_{n} / n \rightarrow 0$ as $n \rightarrow \infty$.

As it can be seen in Section 5.1, $D_{3}\left(u_{n}\right)$ follows, as easily as $D_{2}\left(u_{n}\right)$, from sufficiently fast decay of correlations. As it can be seen in [27] we have the following strengthening of Theorem 1: 
Theorem 4 ([27, Theorem 5]). Let $X_{1}, X_{2}, \ldots$ be a stationary stochastic process for which conditions $D_{3}\left(u_{n}\right)$ and $D^{\prime}\left(u_{n}\right)$ hold for a sequence of levels $u_{n}$ such that (2.3) holds. Then the REPP $N_{n}$ defined in (3.10) is such that $N_{n} \stackrel{d}{\rightarrow} N$, as $n \rightarrow \infty$, where $N$ denotes a Poisson Process with intensity 1.

3.3.2. The presence of clustering. When there is clustering, one cannot use the aforementioned criterion of Kallenberg anymore because the point processes are not simple anymore and possess multiple events. This means that a much deeper analysis must be done in order to obtain convergence of the REPP. We did it in [29] and we describe below the main results and conditions needed. First, we define the sequence $\left(U^{(\kappa)}(u)\right)_{\kappa \geq 0}$ of nested balls centred at $\zeta$ given by: $U^{(0)}(u)=U(u)$ and $U^{(\kappa)}(u)=f^{-p}\left(U^{(\kappa-1)}(u)\right) \cap U(u)$ for all $\kappa \in \mathbb{N}$. For $i, \kappa, \ell, s \in \mathbb{N} \cup\{0\}$, we define the following events: $Q_{p, i}^{\kappa}(u):=f^{-i}\left(U^{(\kappa)}(u)-U^{(\kappa+1)}(u)\right)$.

Observe that for each $\kappa$, the set $Q_{p, 0}^{\kappa}(u)$ corresponds to an annulus centred at $\zeta$. Besides, $U(u)=\bigcup_{\kappa=0}^{\infty} Q_{p, 0}^{\kappa}(u)$, which means that the ball centred at $\zeta$ which corresponds to $U(u)$ can be decomposed into a sequence of disjoint annuli where $Q_{p, 0}^{0}(u)$ is the most outward ring and the inner ring $Q_{p, 0}^{\kappa+1}(u)$ is sent outward by $f^{p}$ to the $\operatorname{ring} Q_{p, 0}^{\kappa}(u)$, i.e., $f^{p}\left(Q_{p, 0}^{\kappa+1}(u)\right)=Q_{p, 0}^{\kappa}(u)$.

We are now ready to state:

Condition $\left(D_{p}\left(u_{n}\right)^{*}\right)$. We say that $D_{p}\left(u_{n}\right)^{*}$ holds for the sequence $X_{0}, X_{1}, X_{2}, \ldots$ if for any integers $t, \kappa_{1}, \ldots, \kappa_{\varsigma}, n$ and any $J=\cup_{i=2}^{\varsigma} I_{j} \in \mathcal{R}$ with $\inf \{x: x \in J\} \geq t$,

$$
\left|\mathbb{P}\left(Q_{p, 0}^{\kappa_{1}}\left(u_{n}\right) \cap\left(\cap_{j=2}^{\varsigma} \mathscr{N}_{u_{n}}\left(I_{j}\right)=\kappa_{j}\right)\right)-\mathbb{P}\left(Q_{p, 0}^{\kappa_{1}}\left(u_{n}\right)\right) \mathbb{P}\left(\cap_{j=2}^{\varsigma} \mathscr{N}_{u_{n}}\left(I_{j}\right)=\kappa_{j}\right)\right| \leq \gamma(n, t),
$$

where for each $n$ we have that $\gamma(n, t)$ is nonincreasing in $t$ and $n \gamma\left(n, t_{n}\right) \rightarrow 0$ as $n \rightarrow \infty$, for some sequence $t_{n}=o(n)$.

This mixing condition is stronger than $D^{p}\left(u_{n}\right)$ because it requires a uniform bound for all $\kappa_{1}$, nonetheless, it still is much weaker than the original $D\left(u_{n}\right)$ from Leadbetter [44] or any of the kind. As all the other preceding conditions $\left(D_{2}, D_{3}, D^{p}\right)$ it can be easily verified for systems with sufficiently fast decay of correlations (see Section 5.1).

In [29], for technical reasons only, we also introduced a slight modification to $D_{p}^{\prime}\left(u_{n}\right)$. The new condition was denoted by $D_{p}^{\prime}\left(u_{n}\right)^{*}$ and is obtained by replacing (3.7) by the following

$$
\lim _{n \rightarrow \infty} n \sum_{j=1}^{\left[n / k_{n}\right]} \mathbb{P}\left(Q_{p, 0}\left(u_{n}\right) \cap\left\{X_{j}>u_{n}\right\}\right)=0 .
$$

We can now state the main theorem in $[29$.

Theorem 5 ( [29, Theorem 1]). Let $X_{0}, X_{1}, \ldots$ be given by (3.1), where $\varphi$ achieves a global maximum at the repelling periodic point $\zeta$, of prime period $p$, and conditions (R1) and (R2) hold. Let $\left(u_{n}\right)_{n \in \mathbb{N}}$ be a sequence satisfying (2.3). Assume that conditions $D_{p}\left(u_{n}\right)^{*}, D_{p}^{\prime}\left(u_{n}\right)^{*}$ hold. Then the REPP $N_{n}$ converges in distribution to a compound Poisson process $N$ with 
intensity $\theta$ and multiplicity d.f. $\pi$ given by $\pi(\kappa)=\theta(1-\theta)^{\kappa}$, for every $\kappa \in \mathbb{N}_{0}$, where the extremal index $\theta$ is given by the expansion rate at $\zeta$ stated in $(\mathrm{R} 2)$.

3.4. Proofs of the existence of EVLs for dynamical systems under the presence and absence of clustering. In this section we will prove Theorems 1,2 and 3 . Since the proofs of Theorems 1 and 3 follow the same lines except for the fact that in the latter the role of the balls is replaced by those of annuli we introduce some notation in order to cope with both simultaneously.

Recall that for every $A \in \mathcal{B}$, we denote the complement of $A$ as $A^{c}:=\mathcal{X} \backslash A$.

Let $A \in \mathcal{B}$ be an event. For some $s \in \mathbb{N} \cup\{0\}$ and $\ell \in \mathbb{N}$, we define:

$$
\mathscr{W}_{s, \ell}(A)=\bigcap_{i=s}^{s+\ell-1} f^{-i}\left(A^{c}\right) .
$$

We will write $\mathscr{W}_{s, \ell}^{c}(A):=\left(\mathscr{W}_{s, \ell}(A)\right)^{c}$. Whenever is clear or unimportant which event $A \in \mathcal{B}$ applies, we will drop the $A$ and write just $\mathscr{W}_{s, \ell}$ or $\mathscr{W}_{s, \ell}^{c}$. Observe that

$$
\mathscr{W}_{0, n}(U(u))=\left\{M_{n} \leq u\right\} \quad \text { and } \quad \mathscr{W}_{0, n}\left(Q_{p}(u)\right)=\mathscr{Q}_{n}(u) .
$$

Lemma 3.1. For any fixed $A \subset \mathcal{B}$ and integers $s, t, m$, with $t<m$, we have:

$$
\left|\mathbb{P}\left(\mathscr{W}_{0, s+t+m}(A)\right)-\mathbb{P}\left(\mathscr{W}_{0, s}(A) \cap \mathscr{W}_{s+t, m}(A)\right)\right| \leq t \mathbb{P}(A)
$$

Proof. Using stationarity we have

$$
\begin{aligned}
\mathbb{P}\left(\mathscr{W}_{0, s} \cap \mathscr{W}_{s+t, m}\right)-\mathbb{P}\left(\mathscr{W}_{0, s+t+m}\right) & =\mathbb{P}\left(\mathscr{W}_{0, s} \cap \mathscr{W}_{s, t}^{c} \cap \mathscr{W}_{s+t, m}\right) \leq \mathbb{P}\left(\mathscr{W}_{0, t}^{c}\right)=\mathbb{P}\left(\cup_{j=0}^{t-1} f^{-j}(A)\right) \\
& \leq \sum_{j=0}^{t-1} \mathbb{P}\left(f^{-j}(A)\right)=t \mathbb{P}(A) .
\end{aligned}
$$

Lemma 3.2. For any fixed $A \subset \mathcal{B}$ and integers $s, t, m$, we have:

$$
\begin{aligned}
& \left|\mathbb{P}\left(\mathscr{W}_{0, s}(A) \cap \mathscr{W}_{s+t, m}(A)\right)-\mathbb{P}\left(\mathscr{W}_{0, m}(A)\right)(1-s \mathbb{P}(A))\right| \leq \\
& \left|s \mathbb{P}(A) \mathbb{P}\left(\mathscr{W}_{0, m}(A)\right)-\sum_{j=0}^{s-1} \mathbb{P}\left(A \cap \mathscr{W}_{s+t-j, m}(A)\right)\right|+2 s \sum_{j=1}^{s-1} \mathbb{P}\left(A \cap f^{-j}(A)\right)
\end{aligned}
$$

Proof. Observe that

$$
\begin{aligned}
\mid \mathbb{P}\left(\mathscr{W}_{0, s} \cap \mathscr{W}_{s+t, m}\right)-\mathbb{P}\left(\mathscr{W}_{0, m}\right) & (1-s \mathbb{P}(A))|\leq| s \mathbb{P}(A) \mathbb{P}\left(\mathscr{W}_{0, m}(A)\right)-\sum_{j=0}^{s-1} \mathbb{P}\left(A \cap \mathscr{W}_{s+t-j, m}(A)\right) \mid+ \\
+ & \left|\mathbb{P}\left(\mathscr{W}_{0, s} \cap \mathscr{W}_{s+t, m}\right)-\mathbb{P}\left(\mathscr{W}_{0, m}\right)+\sum_{j=0}^{s-1} \mathbb{P}\left(A \cap \mathscr{W}_{s+t-j, m}\right)\right|
\end{aligned}
$$


Regarding the second term on the right, by stationarity, we have

$$
\mathbb{P}\left(\mathscr{W}_{0, s} \cap \mathscr{W}_{s+t, m}\right)=\mathbb{P}\left(\mathscr{W}_{s+t, m}\right)-\mathbb{P}\left(\mathscr{W}_{0, s}^{c} \cap \mathscr{W}_{s+t, m}\right)=\mathbb{P}\left(\mathscr{W}_{0, m}\right)-\mathbb{P}\left(\mathscr{W}_{0, s}^{c} \cap \mathscr{W}_{s+t, m}\right) .
$$

Now, since $\mathscr{W}_{0, s}^{c} \cap \mathscr{W}_{s+t, m}=\cup_{i=0}^{s-1} f^{-i}(A) \cap \mathscr{W}_{s+t, m}$, we have

$$
0 \leq \sum_{j=0}^{s-1} \mathbb{P}\left(A \cap \mathscr{W}_{s+t-j, m}\right)-\mathbb{P}\left(\mathscr{W}_{0, s}^{c} \cap \mathscr{W}_{s+t, m}\right) \leq \sum_{j=0}^{s-1} \sum_{i=0, i \neq j}^{s-1} \mathbb{P}\left(f^{-j}(A) \cap f^{-i}(A) \cap W_{s+t, m}\right)
$$

Hence, using these last two computations we get:

$$
\begin{aligned}
\mid \mathbb{P}\left(\mathscr{W}_{0, s} \cap \mathscr{W}_{s+t, m}\right)-\mathbb{P}\left(\mathscr{W}_{0, m}\right)+ & \sum_{j=0}^{s-1} \mathbb{P}\left(A \cap \mathscr{W}_{s+t-j, m}\right) \mid \leq \sum_{j=0}^{s-1} \sum_{i=0, i \neq j}^{s-1} \mathbb{P}\left(f^{-j}(A) \cap f^{-i}(A) \cap \mathscr{W}_{s+t, m}\right) \\
& \leq \sum_{j=0}^{s-1} \sum_{i=0, i \neq j}^{s-1} \mathbb{P}\left(f^{-j}(A) \cap f^{-i}(A)\right) \leq 2 s \sum_{j=1}^{s-1} \mathbb{P}\left(A \cap f^{-j}(A)\right)
\end{aligned}
$$

The result now follows directly from plugging the last estimate into (3.13).

Proposition 3.3. Fix $A \subset \mathcal{B}$ and $n \in \mathbb{N}$. Let $\ell, k \in \mathbb{N}$ be such that $\ell=\lfloor n / k\rfloor$ and $\ell \mathbb{P}(A)<2$. We have:

$$
\begin{aligned}
& \left|\mathbb{P}\left(\mathscr{W}_{0, n}(A)\right)-(1-\ell \mathbb{P}(A))^{k}\right| \leq \\
& 2 k t \mathbb{P}(A)+2 n \sum_{j=1}^{\ell-1} \mathbb{P}\left(A \cap f^{-j}(A)\right)+k\left|\ell \mathbb{P}(A) \mathbb{P}\left(\mathscr{W}_{0, \ell}\right)-\sum_{j=0}^{\ell-1} \mathbb{P}\left(A \cap \mathscr{W}_{\ell+t-j, \ell}\right)\right|
\end{aligned}
$$

Proof. The basic idea is to split the time interval $[0, n)$ into $k$ blocks of size $\lfloor n / k\rfloor$. Then, using Lemma 3.1 we introduce gaps of size $t$ between the blocks, and next we apply Lemma 3.2 recursively until we exhaust all the blocks.

Using Lemma 3.1, with $m=0$, and setting $\mathscr{W}_{i, 0}:=\mathcal{X}$, for all $i=0,1,2, \ldots$, we have:

$$
\left|\mathbb{P}\left(\mathscr{W}_{0, n}\right)-\mathbb{P}\left(\mathscr{W}_{0, k(\ell+t)}\right)\right| \leq k t \mathbb{P}(A) .
$$

It follows by using Lemmas 3.1 and 3.2 that

$$
\begin{aligned}
\mid \mathbb{P}\left(\mathscr{W}_{0, i(\ell+t)}\right)-(1 & -\ell \mathbb{P}(A)) \mathbb{P}\left(\mathscr{W}_{0,(i-1)(\ell+t)}\right)|\leq| \mathbb{P}\left(\mathscr{W}_{0, i(\ell+t)}\right)-\mathbb{P}\left(\mathscr{W}_{0, \ell} \cap \mathscr{W}_{(\ell+t),(i-1)(\ell+t)}\right) \mid \\
& +\left|\mathbb{P}\left(\mathscr{W}_{0, \ell} \cap \mathscr{W}_{(\ell+t),(i-1)(\ell+t)}\right)-(1-\ell \mathbb{P}(A)) \mathbb{P}\left(\mathscr{W}_{0,(i-1)(\ell+t)}\right)\right| \\
\leq & t \mathbb{P}(A)+\left|\ell \mathbb{P}(A) \mathbb{P}\left(\mathscr{W}_{0, \ell}\right)-\sum_{j=0}^{\ell-1} \mathbb{P}\left(A \cap \mathscr{W}_{\ell+t-j, \ell}\right)\right|+2 \ell \sum_{j=1}^{\ell-1} \mathbb{P}\left(A \cap f^{-j}(A)\right),
\end{aligned}
$$

Let $\Upsilon:=t \mathbb{P}(A)+\left|\ell \mathbb{P}(A) \mathbb{P}\left(\mathscr{W}_{0, \ell}\right)-\sum_{j=0}^{\ell-1} \mathbb{P}\left(A \cap \mathscr{W}_{\ell+t-j, \ell}\right)\right|+2 \ell \sum_{j=1}^{\ell-1} \mathbb{P}\left(A \cap f^{-j}(A)\right)$. Since $\ell \mathbb{P}(A)<2$, then it is clear that $|(1-\ell \mathbb{P}(A))|<1$. Also, note that $\left|\mathbb{P}\left(\mathscr{W}_{0, \ell+t}\right)-(1-\ell \mathbb{P}(A))\right| \leq$ 
$\Upsilon$. Now, we use 3.15 recursively to estimate $\left|\mathbb{P}\left(\mathscr{W}_{0, k(\ell+t)}\right)-(1-\ell \mathbb{P}(A))^{k}\right|$. In fact, we have

$$
\begin{aligned}
\left|\mathbb{P}\left(\mathscr{W}_{0, k(\ell+t)}\right)-(1-\ell \mathbb{P}(A))^{k}\right| & \leq \sum_{i=0}^{k-1}(1-\ell \mathbb{P}(A))^{k-1-i}\left|\mathbb{P}\left(\mathscr{W}_{0,(i+1)(\ell+t)}\right)-(1-\ell \mathbb{P}(A)) \mathbb{P}\left(\mathscr{W}_{0, i(\ell+t)}\right)\right| \\
& \leq \sum_{i=0}^{k-1}(1-\ell \mathbb{P}(A))^{k-1-i} \Upsilon \leq k \Upsilon
\end{aligned}
$$

The result follows now at once from $(3.14)$ and 3.16 .

Proof of Theorem 1. Letting $A=U\left(u_{n}\right), \ell=\left\lfloor n / k_{n}\right\rfloor, k=k_{n}$ and $t=t_{n}$ on Proposition 3.3. we obtain

$$
\begin{aligned}
\mid \mathbb{P}\left(M_{n} \leq u_{n}\right)-(1 & \left.-\left\lfloor\frac{n}{k_{n}}\right\rfloor \mathbb{P}\left(U_{n}\right)\right)^{k_{n}} \mid \leq 2 k_{n} t_{n} \mathbb{P}\left(U_{n}\right)+2 n \sum_{j=1}^{\left\lfloor n / k_{n}\right\rfloor-1} \mathbb{P}\left(X_{0}>u_{n} \cap X_{j}>u_{n}\right) \\
& \left.+k_{n}\right\rfloor\left\lfloor\frac{n}{k_{n}}\right\rfloor \mathbb{P}\left(U_{n}\right) \mathbb{P}\left(M_{\left\lfloor n / k_{n}\right\rfloor} \leq u_{n}\right)-\sum_{j=0}^{\left\lfloor n / k_{n}\right\rfloor-1} \mathbb{P}\left(U_{n} \cap \mathscr{W}_{\ell+t-j, \ell}\left(U_{n}\right)\right) \mid .
\end{aligned}
$$

By (2.3) and (2.12), the first term on the right vanishes, as $n \rightarrow \infty$. Condition $D^{\prime}\left(u_{n}\right)$ implies that the second term on the right also goes to 0 , as $n \rightarrow \infty$. Using condition $D_{2}\left(u_{n}\right)$, we have that for the third term:

$$
k_{n}\left\lfloor\left\lfloor\frac{n}{k_{n}}\right\rfloor \mathbb{P}\left(U_{n}\right) \mathbb{P}\left(M_{\left\lfloor n / k_{n}\right\rfloor} \leq u_{n}\right)-\sum_{j=0}^{\left\lfloor n / k_{n}\right\rfloor-1} \mathbb{P}\left(U_{n} \cap \mathscr{W}_{\ell+t-j, \ell}\left(U_{n}\right)\right) \mid \leq n \gamma\left(n, t_{n}\right) \underset{n \rightarrow \infty}{\longrightarrow} 0 .\right.
$$

Finally, by 2.3), we have $\lim _{n \rightarrow \infty}\left(1-\left\lfloor\frac{n}{k_{n}}\right\rfloor \mathbb{P}\left(U_{n}\right)\right)^{k_{n}}=\mathrm{e}^{-\tau}$.

Proof of Theorem 2. Clearly

$$
\left\{M_{n} \leq u_{n}\right\} \subset \mathscr{Q}_{n}\left(u_{n}\right) .
$$

Next, note that if $\mathscr{Q}_{n}\left(u_{n}\right) \backslash\left\{M_{n} \leq u_{n}\right\}$ occurs, then you must enter the ball $\left\{X_{0}>u_{n}\right\}$ at some point which means we may define first time it happens by $i=\inf \{j \in\{0,1, \ldots n-1\}$ : $\left.X_{j}>u_{n}\right\}$ and let $s_{i}=\left[\frac{n-1-i}{p}\right]$. However, since $\mathscr{Q}_{p, 0, n}\left(u_{n}\right)$ does occur, you must never enter the annulus $\left.Q_{(} u_{n}\right)$ which is the only way out of the ball $\left\{X_{0}>u_{n}\right\}$. Hence, once you enter the ball you must never leave it, which means that $f^{-i}\left(\cap_{j=1}^{s_{i}} f^{-j p}\left(X_{0}>u_{n}\right)\right)$ must occur. Consequently,

$$
\mathscr{Q}_{p, 0, n}\left(u_{n}\right) \backslash\left\{M_{n} \leq u_{n}\right\} \subset \bigcup_{i=0}^{n-1} f^{-i}\left(\cap_{j=0}^{s_{i}} f^{-j p}\left(X_{0}>u_{n}\right)\right)
$$


It follows by stationarity, (R2) and (2.3) that

$$
\begin{aligned}
\mathbb{P}\left(\mathscr{Q}_{p, 0, n}\left(u_{n}\right)\right)-\mathbb{P}\left(\left\{M_{n} \leq u_{n}\right\}\right) & \leq \sum_{i=0}^{n-1} \mathbb{P}\left(f^{-i}\left(\cap_{j=0}^{s_{i}} f^{-j p}\left(X_{0}>u_{n}\right)\right)\right) \\
& \leq p \sum_{\kappa=0}^{[n / p]} \mathbb{P}\left(\cap_{j=0}^{\kappa} f^{-j p}\left(X_{0}>u_{n}\right)\right) \\
& \lesssim p \sum_{\kappa=0}^{\infty}(1-\theta)^{\kappa} \mathbb{P}\left(X_{0}>u_{n}\right) \underset{n \rightarrow \infty}{\longrightarrow} 0
\end{aligned}
$$

Remark 3. Observe that condition $S P_{p, \theta}\left(u_{n}\right)$ is designed to obtain the equality in Theorem2 for general stochastic processes not necessarily dynamically defined. As in the previous proof, even without any geometric consideration and appealing only to the definition of $Q_{p}\left(u_{n}\right)$ and $U\left(u_{n}\right)$ in terms of the random variables in the stochastic process $X_{0}, X_{1}, \ldots$, we can easily get to the estimate:

$$
\mathbb{P}\left(\mathscr{Q}_{p, 0, n}\left(u_{n}\right)\right)-\mathbb{P}\left(\left\{M_{n} \leq u_{n}\right\}\right) \leq p \sum_{\kappa=0}^{[n / p]} \mathbb{P}\left(X_{0}>u_{n}, X_{p}>u_{n}, \ldots, X_{\kappa p}>u_{n}\right),
$$

which goes to 0 as $n$ diverges on account of $S P_{p, \theta}\left(u_{n}\right)$.

Proof of Theorem 3. By Remark 3, we have that the first equality in (3.8) holds. We are left then to prove the second equality. Observe that, by definition of $u_{n}, Q_{p}\left(u_{n}\right)$ and (3.5) we have that

$$
n \mathbb{P}\left(Q_{p}\left(u_{n}\right)\right) \sim \theta \tau
$$

Letting $A=Q_{p}\left(u_{n}\right), \ell=\left\lfloor n / k_{n}\right\rfloor, k=k_{n}$ and $t=t_{n}$ on Proposition 3.3, we obtain

$$
\begin{aligned}
& \left|\mathbb{P}\left(\mathscr{Q}_{n}\left(u_{n}\right)\right)-\left(1-\left\lfloor\frac{n}{k_{n}}\right\rfloor \mathbb{P}\left(Q_{p}\left(u_{n}\right)\right)\right)^{k_{n}}\right| \leq 2 k_{n} t_{n} \mathbb{P}\left(Q_{p}\left(u_{n}\right)\right)+2 n \sum_{j=1}^{\left\lfloor n / k_{n}\right\rfloor} \mathbb{P}\left(Q_{p, 0}\left(u_{n}\right) \cap Q_{p, j}\left(u_{n}\right)\right) \\
& \quad+k_{n}\left\lfloor\left\lfloor\frac{n}{k_{n}}\right\rfloor \mathbb{P}\left(Q_{p}\left(u_{n}\right)\right) \mathbb{P}\left(\mathscr{Q}_{0,\left\lfloor n / k_{n}\right\rfloor}\left(u_{n}\right)\right)-\sum_{j=0}^{\left\lfloor n / k_{n}\right\rfloor} \mathbb{P}\left(Q_{p, 0}\left(u_{n}\right) \cap \mathscr{Q}_{\left\lfloor\frac{n}{k_{n}}\right\rfloor+t-j,\left\lfloor\frac{n}{k_{n}}\right\rfloor}\left(u_{n}\right)\right) \mid .\right.
\end{aligned}
$$

By (2.3) and (2.12), the first term on the right vanishes, as $n \rightarrow \infty$. Condition $D_{p}^{\prime}\left(u_{n}\right)$ implies that the second term on the right also goes to 0 , as $n \rightarrow \infty$. Using condition $D^{p}\left(u_{n}\right)$, we have that for the third term:

$$
\begin{array}{r}
k_{n}\left\lfloor\left\lfloor\frac{n}{k_{n}}\right\rfloor \mathbb{P}\left(Q_{p}\left(u_{n}\right)\right) \mathbb{P}\left(\mathscr{Q}_{\left\lfloor n / k_{n}\right\rfloor}\left(u_{n}\right)\right)-\sum_{j=0}^{\left\lfloor n / k_{n}\right\rfloor-1} \mathbb{P}\left(Q_{p, 0}\left(u_{n}\right) \cap \mathscr{Q}_{\left\lfloor\frac{n}{k_{n}}\right\rfloor+t-j,\left\lfloor\frac{n}{k_{n}}\right\rfloor}\left(u_{n}\right)\right) \mid\right. \\
\leq n \gamma^{*}\left(n, t_{n}\right) \underset{n \rightarrow \infty}{\longrightarrow} 0 .
\end{array}
$$


Finally, since by (3.17), we have that $\lim _{n \rightarrow \infty} n \mathbb{P}\left(Q_{p}\left(u_{n}\right)\right)=\theta \tau$ then it follows that $\lim _{n \rightarrow \infty}\left(1-\left\lfloor\frac{n}{k_{n}}\right\rfloor \mathbb{P}\left(Q_{p}\left(u_{n}\right)\right)\right)^{k_{n}}=\mathrm{e}^{-\theta \tau}$.

\section{Hitting Times, Return Times and the link with Extreme Values}

In the dynamical systems setting, another approach to the statistical study of rare events has been addressed since the early works of Pitskel and Hirata [50, 36]. It pertains to the statistical study of the elapsed time before hitting (or returning to) some target sets in the phase space. This type of results is usually referred to as Hitting Time Statistics and Return Time Statistics. In order to define such concepts, we begin by introducing the following notion: consider a set $A \in \mathcal{B}$ and a new r.v. that we refer to as first hitting time to $A$ and denote by $r_{A}: \mathcal{X} \rightarrow \mathbb{N} \cup\{+\infty\}$ where

$$
r_{A}(x)=\min \left\{j \in \mathbb{N} \cup\{+\infty\}: f^{j}(x) \in A\right\} .
$$

for a set.

If there exists a non degenerate d.f. G such that for all $t \geq 0$,

$$
\lim _{u \rightarrow u_{F}} \mathbb{P}\left(r_{U(u)} \leq \frac{t}{\mathbb{P}(U(u))}\right)=G(t),
$$

then we say we have Hitting Time Statistics (HTS) G for balls. Similarly, we can restrict our observations to $U(u)$ : if there exists a non degenerate (d.f.) $\tilde{G}$ such that for all $t \geq 0$,

$$
\lim _{u \rightarrow u_{F}} \mathbb{P}\left(r_{U(u)} \leq \frac{t}{\mathbb{P}(U(u))} \mid U(u)\right)=\tilde{G}(t),
$$

then we say we have Return Time Statistics (RTS) $\tilde{G}$ for balls.

The normalising term in the definition of HTS/RTS is inspired by Kac's Theorem which states that the expected amount of time you have to wait before you return to $U(u)$ is exactly $\frac{1}{\mathbb{P}(U(u))}$.

Remark 4. Let $\mathcal{P}_{0}$ denote a partition of $\mathcal{X}$. We define the corresponding pullback partition $\mathcal{P}_{n}=\bigvee_{i=0}^{n-1} f^{-i}\left(\mathcal{P}_{0}\right)$, where $\vee$ denotes the join of partitions. We refer to the elements of the partition $\mathcal{P}_{n}$ as cylinders of order $n$. For every $\zeta \in \mathcal{X}$, we denote by $\mathrm{Z}_{n}[\zeta]$ the cylinder of order $n$ that contains $\zeta$. For some $\zeta \in \mathcal{X}$ this cylinder may not be unique, but we can make an arbitrary choice, so that $\mathrm{Z}_{n}[\zeta]$ is well defined. We say that the system has HTS (RTS) $G(\tilde{G})$ to cylinders at $\zeta$ if we have HTS (RTS) $G(\tilde{G})$ when $U(u)$ is replaced by the cylinder $\mathrm{Z}_{n}(\zeta)$, and the limit is taken as $n$ diverges.

The existence of exponential HTS $\left(G(t)=1-\mathrm{e}^{-t}\right)$ is equivalent to the existence of exponential RTS $\left(\tilde{G}(t)=1-\mathrm{e}^{-t}\right)$. In fact, according to the Main Theorem in 34, a system has HTS $G$ if and only if it has RTS $\tilde{G}$ and

$$
G(t)=\int_{0}^{t}(1-\tilde{G}(s)) d s .
$$


Note that by assumption (R1) we have that $\left\{M_{n} \leq u\right\}=\left\{r_{U(u)}>n\right\}$. Motivated by this observation, in [27, a complete relation between the existence of HTS/RTS and EVLs was established for absolutely continuous (with respect to Lebesgue) invariant probability measures (acip) and natural observables depending basically on the distance to $\zeta$. Then, in [28], by adapting the observables, this relation was further developed to hold for general equilibrium states.

4.1. The choice of observables. We assume that the observable $\varphi: \mathcal{X} \rightarrow \mathbb{R} \cup\{+\infty\}$ is of the form

$$
\varphi(x)=g\left(\mathbb{P}\left(B_{\operatorname{dist}(x, \zeta)}(\zeta)\right)\right),
$$

where $\zeta$ is a chosen point in the phase space $\mathcal{X}$ and the function $g:[0,+\infty) \rightarrow \mathbb{R} \cup\{+\infty\}$ is such that 0 is a global maximum $(g(0)$ may be $+\infty) ; g$ is a strictly decreasing bijection $g: V \rightarrow W$ in a neighbourhood $V$ of 0 ; and has one of the following three types of behaviour:

Type $g_{1}$ : there exists some strictly positive function $h: W \rightarrow \mathbb{R}$ such that for all $y \in \mathbb{R}$

$$
\lim _{s \rightarrow g_{1}(0)} \frac{g_{1}^{-1}(s+y h(s))}{g_{1}^{-1}(s)}=\mathrm{e}^{-y}
$$

Type $g_{2}: g_{2}(0)=+\infty$ and there exists $\beta>0$ such that for all $y>0$

$$
\lim _{s \rightarrow+\infty} \frac{g_{2}^{-1}(s y)}{g_{2}^{-1}(s)}=y^{-\beta}
$$

Type $g_{3}: g_{3}(0)=D<+\infty$ and there exists $\gamma>0$ such that for all $y>0$

$$
\lim _{s \rightarrow 0} \frac{g_{3}^{-1}(D-s y)}{g_{3}^{-1}(D-s)}=y^{\gamma} \text {. }
$$

It may be shown that no non-degenerate limit applies if $\int_{0}^{g_{1}(0)} g_{1}^{-1}(s) d s$ is not finite. Hence, an appropriate choice of $h$ in the Type 1 case is given by $h(s)=\int_{s}^{g_{1}(0)} g_{1}^{-1}(t) d t / g_{1}^{-1}(s)$ for $s<g_{1}(0)$.

Examples of each one of the three types are as follows: $g_{1}(x)=-\log x$ (in this case (4.3) is easily verified with $h \equiv 1), g_{2}(x)=x^{-1 / \alpha}$ for some $\alpha>0$ (condition (4.4) is verified with $\beta=\alpha$ ) and $g_{3}(x)=D-x^{1 / \alpha}$ for some $D \in \mathbb{R}$ and $\alpha>0$ (condition (4.5) is verified with $\gamma=\alpha)$.

In [27] we assumed that $\varphi(x)=g(\operatorname{dist}(x, \zeta))$. Since the invariant measure there was an acip, using Lebesgue's differentiation theorem, we could write $\mathbb{P}\left(B_{\eta}(\zeta)\right) \sim \rho(\zeta) \operatorname{Leb}\left(B_{\eta}(\zeta)\right)$, where we assume that $\rho(\zeta)=\frac{d \mathbb{P}}{\mathrm{Leb}}(\zeta)>0$ and Lebesgue's differentiation theorem applies to $\zeta$. In here, since $\mathbb{P}$ may not be an acip the function $\hbar$ defined for small $\eta \geq 0$ and given by

$$
\hbar(\eta)=\mathbb{P}\left(B_{\eta}(\zeta)\right)
$$

may not be absolutely continuous. However, we require that $\hbar$ is continuous in $\eta$. For example, if $\mathcal{X}$ is an interval and $\mathbb{P}$ a Borel probability with no atoms,i.e., points with 
positive $\mathbb{P}$ measure, then $\hbar$ is continuous. One of our applications is to equilibrium states, which we explain in Section 5.3. A major difference here is that although $g$ is invertible in a small neighbourhood of 0 , the function $\hbar$ does not have to be. This means that, in contrast with [27], the observable $\varphi$, as a function of the distance to $\zeta$, may not be invertible in any small neighbourhood of $\zeta$.

For that reason, we now set

$$
\ell(\gamma):=\inf \left\{\eta>0: \mathbb{P}\left(B_{\eta}(\zeta)\right)=\gamma\right\}
$$

which is well defined for all small enough $\gamma \geq 0$, by the continuity of $\hbar$. Moreover, again by continuity of $\hbar$, we have

$$
\mathbb{P}\left(B_{\ell(\gamma)}(\zeta)\right)=\gamma
$$

Remark 5. Observe that the choice of the observables in $(4.2)$ and the assumption on $\mathbb{P}$ regarding the continuity of $\hbar$ guarantee that condition (R1) holds.

Remark 6. Observe that if at time $j \in \mathbb{N}$ we have an exceedance of level $u$ (sufficiently large), i.e., $X_{j}(x)>u$, then we have an entrance of the orbit of $x$ into the ball $B_{\ell\left(g^{-1}(u)\right)}(\zeta)$ of radius $\ell\left(g^{-1}(u)\right)$ around $\zeta$, at time $j$. This means that the behaviour of the tail of $F$, i.e., the behaviour of $1-F(u)$ as $u \rightarrow u_{F}$ is basically determined by $g^{-1}$. The above conditions are just the translation in terms of the shape of $g^{-1}$, of the sufficient and necessary conditions on the tail of $F$ that appear in $(2.9),(2.10)$ and $(2.11)$.

The fact that the conditions on the shape of $g^{-1}$ imposed by 4.3$),(4.4)$ and $(4.5)$ correspond to the sufficient and necessary conditions (2.9), 2.10) and (2.11), respectively, on the tail of a distribution which guarantee a non-degenerate EVL in the iid setting, means that the only interesting cases for us are the ones where $g$ is of one of the three types above.

Remark 7. The choice of the observables in 4.2 implies that the shape of $g$ determines the type of extremal distribution we get. In particular, for observables of type $g_{i}$ we get an extremal law of type $\mathrm{e}^{-\tau_{i}}$, for $i=1,2,3$. While the type of the extremal distribution is essentially determined by the shape of the observable, in the cases when types 2 and 3 apply, i.e., the Fréchet and Weibull families of distributions, respectively, the exponent $\alpha$ in (2.8) is also influenced by other quantities such as the EI and the local dimension of the stationary invariant measure $\mathbb{P}$. In particular, when such measure is absolutely continuous with respect to Lebesgue and its Radon-Nikodym derivative has a singularity at $\zeta$, then the order of the singularity also influences the value of $\alpha$.

4.2. The connection between EVL and HTS. For the statements below, we recall that the stochastic process $X_{0}, X_{1}, \ldots$ is defined by (3.1) where the observable $\varphi$ is defined by (4.2). We stress that $g$ stands for some $g_{i}$ with $i=1,2,3$ defined by conditions (4.3), (4.4) and 4.5), respectively.

Our first main result, which obtains EVLs from HTS for balls, is the following.

Theorem 6 ([28, Theorem 1]). Let $(\mathcal{X}, \mathcal{B}, \mathbb{P}, f)$ be a dynamical system, $\zeta \in \mathcal{X}$ be in the support of $\mathbb{P}$ and assume that $\mathbb{P}$ is such that the function $\hbar$ defined on (4.6) is continuous. 
If we have HTS $G$ to balls centred on $\zeta \in \mathcal{X}$, then we have an $E V L H=G$ for $M_{n}$ that applies to the observables 4.2 achieving a maximum at $\zeta$. Moreover, for all $i=1,2,3$, if $u_{n}$ is chosen as linear normalising sequence, as in (2.6), then the shape $g_{i}$ for the observable corresponds to an extremal type law of the form $e^{-\tau_{i}}$, given in (2.8).

Now, we state a result in the other direction, i.e., we show how to get HTS from EVLs for balls.

Theorem 7 ([28, Theorem 2]). Let $(\mathcal{X}, \mathcal{B}, \mathbb{P}, f)$ be a dynamical system, $\zeta \in \mathcal{X}$ be in the support of $\mathbb{P}$ and assume that $\mathbb{P}$ is such that the function $\hbar$ defined in (4.6) is continuous.

If we have an EVL $H$ for $M_{n}$ which applies to the observables 4.2 achieving a maximum at $\zeta \in \mathcal{X}$ then we have HTS $G=H$ to balls at $\zeta$.

\section{Existence of Extreme Value Laws for Chaotic systems}

As it can be seen in Section 4, the theory of HTS/RTS and the theory of EVL for dynamical systems are two sides of the same coin. This means that we can prove the existence of EVLs by proving HTS and the other way around.

The theory of HTS/RTS laws is now a well developed topic, applied first to cylinders and hyperbolic dynamics, and then extended to balls and also to non-uniformly hyperbolic systems. We refer to [14] and [53] for very nice reviews as well as many references on the subject. (See also [3], where the focus is more towards a finer analysis of uniformly hyperbolic systems.) Since the early papers [50, 36, several different approaches have been used to prove HTS/RTS: from the analysis of adapted Perron-Frobenius operators as in [36], the use of inducing schemes as in [10], to the relation between recurrence rates and dimension as explained in [53, Section 4].

For many mixing systems it is known that the HTS/RTS are standard exponential around almost every point. Among these systems we note the following: Markov chains [50, Axiom A diffeomorphisms [36], uniformly expanding maps of the interval [15], 1-dimensional nonuniformly expanding maps [37, 10, 12, 11, partially hyperbolic dynamical systems [18, toral automorphisms [17], higher dimensional non-uniformly hyperbolic systems (including Hénon maps) [13.

In most of the papers mentioned so far, exponential HTS and RTS have been proved for generic points, in the sense that there exists exponential HTS/RTS for almost all $\zeta$ in the phase space, with respect to the invariant measure. However, in [36] and [35], the authors consider specific points. Namely, they consider the cases when $\zeta$ is a periodic point and obtain the existence of an EI less than 1, although they did not stated in these terms because, at the time, the connection with EVL was not yet established. In fact, Galves and Schmitt [31] introduced a short correction factor $\lambda$ in order to get exponential HTS, that was then studied later in great detail by Abadi et al. [3, 1, 2, 5, 6, 4, and which, in case of being convergent, can actually be seen as the EI itself. 
On the other hand, EVLs for the partial maximum of dynamically defined stochastic processes is a much recent topic and have been proved directly in the recent papers [16, 25, 27, 38, 33, 28, 30, 29, 41, 8]. We highlight the pioneering work of Collet [16] for the innovative ideas introduced. The dynamical systems covered in these papers include non-uniformly hyperbolic 1-dimensional maps (in all of them), higher dimensional nonuniformly expanding maps in [27], suspension flows in [38], billiards and Lozi maps in [33]. In [8], using the advantage of the simplicity of the EVL approach, in terms of its definition from a stationary stochastic process, it is shown that for randomly perturbed systems with additive noise one can prove exponential HTS/RTS and EVLs, regardless of the point $\zeta$ chosen in the phase space.

In this section, we will prove the existence of EVLs for uniformly expanding and piecewise expanding systems. Although, these are not as general as the non-uniformly expanding systems treated in many of the papers mentioned above, for these systems we can actually prove a dichotomy which basically states that either there exists an EI less than 1 at periodic repelling points or there exists an EI equal to 1 at every other point of continuity of the map.

Up to our knowledge, the statement of this dichotomy appeared first in [30, Section 6], where it was proved for uniformly expanding systems in $S^{1}$ equipped with the Bernoulli measure and for the cylinder case. Moreover, in the introduction of [30], it was conjectured that this dichotomy should hold in much greater generality (both for more general systems and for the more general case of balls rather than cylinders). In [23], which appeared shortly after [30] on arXiv, the authors build up on the work of [36] and eventually obtain the dichotomy for balls and for conformal repellers. Then, in [41], making use of powerful spectral theory tools developed in [42], the dichotomy for balls was established for general systems such as those for which there exists as spectral gap for their respective Perron-Frobenius operator. In [8], the dichotomy for balls was obtained once again for the same type of systems considered in [41] but using as assumption the existence of decay of correlations against $L^{1}$ observables (see definition below). Finally, in the very recent [43], the dichotomy for cylinders is established for mixing countable alphabet shifts, but also in the context of nonconventional ergodic sums.

Our basic assumption to prove conditions $D_{2}\left(u_{n}\right), D^{\prime}\left(u_{n}\right)$ and their twins $D^{p}\left(u_{n}\right), D_{p}^{\prime}\left(u_{n}\right)$, will be sufficiently fast decay of correlations, in some specific function spaces. Hence we define:

Definition 5 (Decay of correlations). Let $\mathcal{C}_{1}, \mathcal{C}_{2}$ denote Banach spaces of real valued measurable functions defined on $\mathcal{X}$. We denote the correlation of non-zero functions $\phi \in \mathcal{C}_{1}$ and $\psi \in \mathcal{C}_{2}$ w.r.t. a measure $\mathbb{P}$ as

$$
\operatorname{Cor}_{\mathbb{P}}(\phi, \psi, n):=\frac{1}{\|\phi\|_{\mathcal{C}_{1}}\|\psi\|_{\mathcal{C}_{2}}}\left|\int \phi\left(\psi \circ f^{n}\right) \mathrm{d} \mathbb{P}-\int \phi \mathrm{d} \mathbb{P} \int \psi \mathrm{d} \mathbb{P}\right|
$$


We say that we have decay of correlations, w.r.t. the measure $\mathbb{P}$, for observables in $\mathcal{C}_{1}$ against observables in $\mathcal{C}_{2}$ if, for every $\phi \in \mathcal{C}_{1}$ and every $\psi \in \mathcal{C}_{2}$ we have

$$
\mathrm{Cor}_{\mathbb{P}}(\phi, \psi, n) \rightarrow 0, \quad \text { as } n \rightarrow \infty \text {. }
$$

We say that we have decay of correlations against $L^{1}$ observables whenever this holds for $\mathcal{C}_{2}=L^{1}(\mathbb{P})$ and $\|\psi\|_{\mathcal{C}_{2}}=\|\psi\|_{1}=\int|\psi| \mathrm{d} \mathbb{P}$.

We are now in condition of stating an abstract result giving general conditions to establish the dichotomy:

Theorem 8 ([8]). Consider a continuous dynamical system $(\mathcal{X}, \mathcal{B}, \mathbb{P}, f)$ for which there exists a Banach space $\mathcal{C}$ of real valued functions such that for all $\phi \in \mathcal{C}$ and $\psi \in L^{1}(\mathbb{P})$,

$$
\operatorname{Cor}_{\mathbb{P}}(\phi, \psi, n) \leq C n^{-2},
$$

where $C>0$ is a constant independent of both $\phi, \psi$. Let $X_{0}, X_{1}, \ldots$ be given by (3.1), where $\varphi$ achieves a global maximum at some $\zeta \in \mathcal{X}$ and condition (R1) holds.

- If $\zeta$ is a non periodic point and there exists some $C>0$ such that, for all $n \in \mathbb{N}$, we have $\left\|\mathbf{1}_{U\left(u_{n}\right)}\right\|_{\mathcal{C}} \leq C$, then conditions $D_{2}\left(u_{n}\right)$ and $D^{\prime}\left(u_{n}\right)$ hold for $X_{0}, X_{1}, \ldots$ which means we have an EI equal to 1, i.e., we have an EVL with $H(\tau)=1-\mathrm{e}^{-\tau}$.

- If $\zeta$ is a periodic point of prime period $p$, at which condition (R2) holds and there exists some $C>0$ such that, for all $n \in \mathbb{N}$, we have $\left\|\mathbf{1}_{Q_{p}\left(u_{n}\right)}\right\|_{\mathcal{C}} \leq C$, then conditions $D^{p}\left(u_{n}\right)$ and $D_{p}^{\prime}\left(u_{n}\right)$ hold for $X_{0}, X_{1}, \ldots$ which means we have an EI equal to $\theta<1$, i.e., we have an $E V L$ with $H(\tau)=1-\mathrm{e}^{-\theta \tau}$, where $\theta$ is given in $(\mathrm{R} 2)$.

In fact, if we consider the REPP we can also state the following stronger version of the dichotomy

Theorem 9 ([8]). Under the same assumptions of Theorem 8

- If $\zeta$ is a non periodic point and there exists some $C>0$ such that, for all $n \in \mathbb{N}$, we have $\left\|\mathbf{1}_{U\left(u_{n}\right)}\right\|_{\mathcal{C}} \leq C$, then conditions $D_{3}\left(u_{n}\right)$ and $D^{\prime}\left(u_{n}\right)$ hold for $X_{0}, X_{1}, \ldots$ which means the REPP $N_{n}$ defined in (3.10) converges in distribution, as $n \rightarrow \infty$ to a standard Poisson process.

- If $\zeta$ is a periodic point of prime period p, at which condition (R2) holds and there exists some $C>0$ such that, for all $n \in \mathbb{N}$, we have $\left\|\mathbf{1}_{Q_{p}\left(u_{n}\right)}\right\|_{\mathcal{C}} \leq C$, then conditions $D^{p}\left(u_{n}\right)^{*}$ and $D_{p}^{\prime}\left(u_{n}\right)^{*}$ hold for $X_{0}, X_{1}, \ldots$ which means that the REPP $N_{n}$ converges in distribution to a compound Poisson process $N$ with intensity $\theta$ and multiplicity d.f. $\pi$ given by $\pi(\kappa)=\theta(1-\theta)^{\kappa}$, for every $\kappa \in \mathbb{N}_{0}$, where the extremal index $\theta$ is given by the expansion rate at $\zeta$ stated in $(\mathrm{R} 2)$.

Remark 8. Observe that decay of correlations as in (5.1) against $L^{1}(\mathbb{P})$ observables is a very strong property. In fact, regardless of the rate (in this case $n^{-2}$ ), as long as it is summable, one can actually show that the system has exponential decay of correlations of Hölder observables against $L^{\infty}(\mathbb{P})$. See [7, Theorem B]. 
Remark 9. For simplicity, here, we will not deal with discontinuity points of the map $f$. However, that can be done and the existence of an EI less than 1 depends on the existence of some periodic behaviour. Otherwise, we also get an EI equal to 1. See [8, Section 3.3].

Remark 10. We observe that the second statement of Theorem 8 had already been established in [30], while the second statement of Theorem 9 had already been established in [29, Theorem 2]. The first statements of Theorem 8 and 9 , which finally allowed to establish the dichotomy, were obtained in [8].

In Section 5.3 we will show some specific expanding and piecewise expanding systems for which we can verify the assumptions in Theorems 8 and 9 but to illustrate the essence of their content, we give here a straightforward application to a very simple system: the doubling map.

Corollary 10. Let $S^{1}:=\{z \in \mathcal{C}:|z|=1\}$ with the usual metric that we denote by dist. Consider the system $f: S^{1} \rightarrow S^{1}$ given by $f(z)=z^{2}$, equipped with the Haar (or Lebesgue) measure. Let $X_{0}, X_{1}, \ldots$ be given by (3.1), where $\varphi$ achieves a global maximum at some $\zeta \in S^{1}$ (Take for example $\varphi(z)=1-\operatorname{dist}(z, \zeta)$ ). Then,

- If $\zeta \in S^{1}$ is not periodic then the EI is equal to 1 and the REPP $N_{n}$ defined in (3.10) converges in distribution as $n \rightarrow \infty$ to a standard Poisson process.

- If $\zeta \in S^{1}$ is periodic of prime period $p \in \mathbb{N}$ then the EI is equal to $\theta=1-(1 / 2)^{p}$ and the REPP $N_{n}$ defined in (3.10) converges in distribution as $n \rightarrow \infty$ to a compound Poisson process $N$ with intensity $\theta$ and multiplicity d.f. $\pi$ given by $\pi(\kappa)=\theta(1-\theta)^{\kappa}$, for every $\kappa \in \mathbb{N}_{0}$.

5.1. Conditions $D_{2}\left(u_{n}\right), D_{3}\left(u_{n}\right), D^{p}\left(u_{n}\right), D^{p}\left(u_{n}\right)^{*}$ and decay of correlations. Rates of decay of correlations are nowadays well known for many chaotic systems. Examples of these include hyperbolic or uniformly expanding systems as well as the non hyperbolic or non-uniformly expanding admitting, for example, inducing schemes with a well behaved return time function. In fact, in two remarkable papers Lai-Sang Young showed that the rates of decay of correlations of the original system are intimately connected with the recurrence rates of the respective induced map.

In general terms the conditions $D_{2}\left(u_{n}\right), D_{3}\left(u_{n}\right), D^{p}\left(u_{n}\right)$ and $D^{p}\left(u_{n}\right)^{*}$ follow from sufficiently fast (e.g. polynomial) decay of correlations of the dynamical system. This is where they are seen to be much more useful than Leadbetter's $D\left(u_{n}\right)$. While $D\left(u_{n}\right)$ usually follows only from strong uniform mixing, like $\alpha$-mixing (see [9] for definition), and even then only at certain subsequences, which means most of the time the final result holds only for cylinders, $D_{2}\left(u_{n}\right), D_{3}\left(u_{n}\right), D^{p}\left(u_{n}\right)$ and $D^{p}\left(u_{n}\right)^{*}$ follow from decay of correlations which is much weaker and allows to obtain the result for balls, instead.

In fact, in order to prove that $D_{2}\left(u_{n}\right), D_{3}\left(u_{n}\right), D^{p}\left(u_{n}\right)$ and $D^{p}\left(u_{n}\right)^{*}$, there is actually no need for such strong type of decay of correlations as such as against $L^{1}$, like in the assumptions of Theorems 8 or 9 . It suffices to have decay of correlations against all $\psi$ in $L^{\infty}$, for example. 
Just to give an idea of how simple it is to check $D_{2}\left(u_{n}\right), D_{3}\left(u_{n}\right), D^{p}\left(u_{n}\right)$ and $D^{p}\left(u_{n}\right)^{*}$ for systems with sufficiently fast decay of correlations, let us begin by defining the following Banach spaces:

Given a function $\psi: Y \rightarrow \mathbb{R}$ on an interval $Y$, the variation of $\psi$ is defined as

$$
\operatorname{Var}(\psi):=\sup \left\{\sum_{i=0}^{n-1}\left|\psi\left(x_{i+1}\right)-\psi\left(x_{i}\right)\right|\right\},
$$

where the supremum is taken over all finite ordered sequences $\left(x_{i}\right)_{i=0}^{n} \subset Y$.

We use the norm $\|\psi\|_{B V}=\sup |\psi|+\operatorname{Var}(\psi)$, which makes $B V:=\left\{\psi: Y \rightarrow \mathbb{R}:\|\psi\|_{B V}<\infty\right\}$ into a Banach space.

Now, let $\mathcal{X}$ be a compact subset of $\mathbb{R}^{d}$ and let $\psi: \mathcal{X} \rightarrow \mathbb{R}$. Given a Borel set $\Gamma \subset \mathcal{X}$, we define the oscillation of $\psi \in L^{1}$ (Leb) over $\Gamma$ as

$$
\operatorname{osc}(\psi, \Gamma):=\underset{\Gamma}{\operatorname{ess} \sup } \psi-\underset{\Gamma}{\operatorname{ess} \inf } \psi \text {. }
$$

It is easy to verify that $x \mapsto \operatorname{osc}\left(\psi, B_{\varepsilon}(x)\right)$ defines a measurable function (see [52, Proposition 3.1]). Given real numbers $0<\alpha \leq 1$ and $\varepsilon_{0}>0$, we define $\alpha$-seminorm of $\psi$ as

$$
|\psi|_{\alpha}=\sup _{0<\varepsilon \leq \varepsilon_{0}} \varepsilon^{-\alpha} \int_{\mathbb{R}^{d}} \operatorname{osc}\left(\psi, B_{\varepsilon}(x)\right) \mathrm{dLeb}(x) .
$$

Let us consider the space of functions with bounded $\alpha$-seminorm $V_{\alpha}=\left\{\psi \in L^{1}(\mathrm{Leb})\right.$ : $\left.|\psi|_{\alpha}<\infty\right\}$, and endow $V_{\alpha}$ with the norm $\|\cdot\|_{\alpha}=\|\cdot\|_{L^{1}(\mathrm{Leb})}+|\cdot|_{\alpha}$ which makes it into a Banach space. We note that $V_{\alpha}$ is independent of the choice of $\varepsilon_{0}$.

In what follows we assume that for every $\zeta \in \mathcal{X}$ condition (R1) holds and, in case $\zeta$ is periodic of period $p$ then condition (R2) also holds.

Proposition 5.1. Assume that for our system $f: \mathcal{X} \rightarrow \mathcal{X}$ we have decay of correlations for all $\phi \in \mathcal{C}$, where $\mathcal{C}$ is $B V$ or $V_{\alpha}$, depending on whether $\mathcal{X}$ is a compact subset of $\mathbb{R}$ or $\mathbb{R}^{d}$ (with $\left.d=2,3, \ldots\right)$, and all $\psi \in L^{\infty}$ so that there exist $C$, independent of $\phi, \psi$, and a rate function $\varrho: \mathbb{N} \rightarrow \mathbb{R}$ such that

$$
\left|\int \phi \cdot\left(\psi \circ f^{t}\right) d \mathbb{P}-\int \phi d \mathbb{P} \int \psi d \mathbb{P}\right| \leq C\|\phi\|_{\mathcal{C}}\|\psi\|_{\infty} \varrho(t), \quad \forall t \in \mathbb{N}_{0},
$$

and $n \varrho\left(t_{n}\right) \rightarrow 0$, as $n \rightarrow \infty$ for some $t_{n}=o(n)$. Then conditions $D_{2}\left(u_{n}\right), D_{3}\left(u_{n}\right), D^{p}\left(u_{n}\right)$ and $D^{p}\left(u_{n}\right)^{*}$ hold.

Proof. In what follows, for all $n \in \mathbb{N}$, let $A_{n}=U\left(u_{n}\right)$, in case $\zeta$ is non periodic, and $A_{n}=Q_{p}\left(u_{n}\right)$ if $\zeta$ is periodic of prime period $p$. Take $\phi=\mathbf{1}_{A_{n}}, \psi=\mathbf{1}_{\mathscr{W}_{0, \ell}\left(A_{n}\right)}$. Observe that whether $\|\cdot\|_{\mathcal{C}}$ is $\|\cdot\|_{B V}$ or $\|\cdot\|_{\alpha}$, we have that there exists some $C^{\prime}>0$ such that $\left\|\mathbf{1}_{A_{n}}\right\|_{\mathcal{C}} \leq C^{\prime}$, for all $n \in \mathbb{N}$. Set $c=C C^{\prime}$. Then $(5.2)$ implies that, in case $\zeta$ is non periodic, condition $D_{2}\left(u_{n}\right)$ holds and, in case $\zeta$ is periodic of prime period $p$, condition $D^{p}\left(u_{n}\right)$ holds, where $\gamma(n, t)=\gamma(t):=c \varrho(t)$ and for the sequence $t_{n}$ such that $n \varrho\left(t_{n}\right) \rightarrow 0$, as $n \rightarrow \infty$. 
Note that to prove $D_{3}\left(u_{n}\right)$, we just have to take $\psi=\mathbf{1}_{\mathscr{N}(A)=0}$ and for $D^{p}\left(u_{n}\right)^{*}$, we would take $\phi=\mathbf{1}_{Q_{p, 0}^{\kappa_{1}\left(u_{n}\right)}}, \psi=\mathbf{1}_{\left(\cap_{j=2}^{\varsigma} \mathscr{N}_{u_{n}}\left(I_{j}\right)=\kappa_{j}\right)}$ and the argument would follow as before.

Note that, in the proof above, to check $D_{2}\left(u_{n}\right)$ and $D^{p}\left(u_{n}\right)$, it was useful the facts that $\mathbf{1}_{A_{n}} \in \mathcal{C}$ and $\left\|\mathbf{1}_{A_{n}}\right\|_{\mathcal{C}} \leq C^{\prime}$. However, these conditions can still be checked even when $\mathbf{1}_{A_{n}} \notin \mathcal{C}$. This is the case when $\mathcal{C}$ is the Banach space of Hölder observables which is used, for example, to obtain decay of correlations for systems with Young towers. The idea is to use, as in [16, Lemma 3.3], an adequate Hölder approximation for $\mathbf{1}_{A_{n}}$.

Now, let $\mathcal{X}$ be a compact subset of $\mathbb{R}^{d}$ and let $\phi: \mathcal{X} \rightarrow \mathbb{R}$. Let $\mathcal{H}_{\beta}$ denote the space of Hölder continuous functions $\phi$ with exponent $\beta$ equipped with the norm $\|\phi\|_{\mathcal{H}_{\beta}}=\|\phi\|_{\infty}+|\phi|_{\mathcal{H}_{\beta}}$, where

$$
|\phi|_{\mathcal{H}_{\beta}}=\sup _{x \neq y} \frac{|\phi(x)-\phi(y)|}{|x-y|^{\beta}} .
$$

Proposition 5.2. Assume that $\mathcal{X}$ is a compact subset of $\mathbb{R}^{d}$ and $f: \mathcal{X} \rightarrow \mathcal{X}$ is a system with an acip $\mathbb{P}$, such that $\frac{d \mathbb{P}}{\mathrm{Leb}} \in L^{1+\epsilon}$. Assume, moreover, that the system has decay of correlations for all $\phi \in \mathcal{H}_{\beta}$ against any $\psi \in L^{\infty}$ so that there exists some $C>0$ independent of $\phi, \psi$ and $t$, and a rate function $\varrho: \mathbb{N} \rightarrow \mathbb{R}$ such that

$$
\left|\int \phi \cdot\left(\psi \circ f^{t}\right) d \mathbb{P}-\int \phi d \mathbb{P} \int \psi d \mathbb{P}\right| \leq C\|\phi\|_{\mathcal{H}_{\beta}}\|\psi\|_{\infty} \varrho(t),
$$

and $n^{1+\beta(1+\max \{(0, \epsilon+1) / \epsilon-d\}+\delta)} \varrho\left(t_{n}\right) \rightarrow 0$, as $n \rightarrow \infty$ for some $\delta>0$ and $t_{n}=o(n)$. Then conditions $D_{2}\left(u_{n}\right), D_{3}\left(u_{n}\right), D^{p}\left(u_{n}\right)$ and $D^{p}\left(u_{n}\right)^{*}$ hold.

Proof. Since $\frac{d \mathbb{P}}{\mathrm{Leb}} \in L^{1+\epsilon}$, by Hölder's inequality, this last fact implies that for all Borel sets $B$, there exists $C>0$ such that

$$
\mathbb{P}(B) \leq C(\operatorname{Leb}(B))^{\Theta},
$$

where $\Theta=\epsilon /(1+\epsilon)$.

For $\eta=\max \left\{\Theta^{-1}-d, 0\right\}+\delta>0$ we build the Hölder approximation $\phi$ of $\mathbf{1}_{A_{n}}$, where $A_{n}$ is as in the proof of Proposition 5.1. Let $D_{n}:=\left\{x \in A_{n}: \operatorname{dist}\left(x, \overline{A_{n}^{c}}\right) \geq\left(\mathbb{P}\left(A_{n}\right)\right)^{1+\eta}\right\}$, where $\bar{A}$ denotes the closure of $A$. Define $\phi: \mathcal{X} \rightarrow \mathbb{R}$ as

$$
\phi(x)= \begin{cases}0 & \text { if } x \in \overline{A_{n}^{c}} \\ \frac{\operatorname{dist}\left(x, \overline{A_{n}^{c}}\right)}{\operatorname{dist}\left(x, \overline{A_{n}^{c}}\right)+\operatorname{dist}\left(x, D_{n}\right)} & \text { if } x \in{\overline{A_{n}^{c}}}^{c} \backslash D_{n} . \\ 1 & \text { if } x \in D_{n}\end{cases}
$$

Observe that $\phi$ is Hölder continuous with Hölder constant $\left(\mathbb{P}\left(A_{n}\right)\right)^{-\beta(1+\eta)}$.

Now, we apply the decay of correlations to the Hölder continuous function $\phi$ against $\psi=$ $\mathbf{1}_{\mathscr{W}_{0, \ell}\left(A_{n}\right)}$ to get

$$
\left|\int \phi \cdot\left(\mathbf{1}_{\mathscr{W}_{0, \ell}\left(A_{n}\right)} \circ f^{t}\right) d \mathbb{P}-\int \phi d \mathbb{P} \int \mathbf{1}_{\mathscr{W}_{0, \ell}\left(A_{n}\right)} d \mathbb{P}\right| \leq C\left(\mathbb{P}\left(A_{n}\right)\right)^{-\beta(1+\eta)} \varrho(t) .
$$


Observe that the support of $\mathbf{1}_{A_{n}}-\phi$ is contained in $A_{n} \backslash D_{n}$ whose Lebesgue measure is $O\left(\left(\mathbb{P}\left(A_{n}\right)\right)^{d+\eta}\right)$ and, using $(5.4)$, we get that $\int \mathbf{1}_{A_{n}}-\phi d \mathbb{P} \leq O\left(\left(\mathbb{P}\left(A_{n}\right)\right)^{\Theta(d+\eta)}\right)$. It follows that

$$
\left|\mathbb{P}\left(A_{n} \cap f^{-t}\left(\mathscr{W}_{0, \ell}\left(A_{n}\right)\right)\right)-\mathbb{P}\left(A_{n}\right) \mathbb{P}\left(\mathscr{W}_{0, \ell}\left(A_{n}\right)\right)\right| \leq\left(\mathbb{P}\left(A_{n}\right)\right)^{-\beta(1+\eta)} \varrho(t)+O\left(\left(\mathbb{P}\left(A_{n}\right)\right)^{\Theta(d+\eta)}\right) .
$$

Hence, we take $\gamma(n, t)=O\left(\left(\mathbb{P}\left(A_{n}\right)\right)^{-\beta(1+\eta)}\right) \varrho(t)+O\left(\left(\mathbb{P}\left(A_{n}\right)\right)^{\Theta(d+\eta)}\right)$. Let $t_{n}$ be as in the hypothesis and recalling that $\mathbb{P}\left(A_{n}\right) \sim \theta \tau / n$, where $\theta<1$ is given by (R2) if $\zeta$ is periodic and $\theta=1$ otherwise, we have that $n \gamma\left(n, t_{n}\right) \leq O\left(n^{1+\beta(1+\eta)}\right) \varrho\left(t_{n}\right)+O\left(n^{-\delta}\right) \underset{n \rightarrow \infty}{\longrightarrow} 0$. As before, to prove $D_{3}\left(u_{n}\right)$ the argument is the same except for the fact that we need to take $\psi=\mathbf{1}_{\mathscr{N}(A)=0}$. In order to prove $D^{p}\left(u_{n}\right)^{*}$ we just need to follow the proof as before and use a Hölder continuous approximation for $\mathbf{1}_{Q_{p}^{\kappa_{1}}\left(u_{n}\right)}$. The only extra difficulty is that we need an upper bound that works for all $\kappa_{1} \in \mathbb{N}_{0}$. Now, taking $\psi=\mathbf{1}_{\left(\cap_{j=2}^{\varsigma} \mathscr{N}_{u_{n}}\left(I_{j}\right)=\kappa_{j}\right)}$, recalling that $\mathbb{P}\left(Q_{p, 0}^{\kappa}\right) \sim \theta(1-\theta)^{\kappa} \mathbb{P}\left(X_{0}>u_{n}\right)$ and following the same footsteps, we easily conclude that (5.3) leads to the following estimate

$$
\begin{aligned}
& \mid \mathbb{P}\left(Q_{p, 0}^{\kappa_{1}}\left(u_{n}\right) \cap\left(\cap_{j=2}^{\varsigma} \mathscr{N}_{u_{n}}\left(I_{j}\right)=\kappa_{j}\right)\right)-\mathbb{P}\left(Q_{p, 0}^{\kappa_{1}}\left(u_{n}\right)\right) \mathbb{P}\left(\cap_{j=2}^{\varsigma} \mathscr{N}_{u_{n}}\left(I_{j}\right)=\kappa_{j}\right) \mid \\
& \leq C\left(\left(\frac{n}{(1-\theta)^{\kappa_{1}}}\right)^{\beta(1+\eta)} \varrho(t)+\left(\frac{(1-\theta)^{\kappa_{1}}}{n}\right)^{\Theta(d+\eta)}\right),
\end{aligned}
$$

for any $\eta>0$, some $C>0$ and where $d$ is the dimension of $\mathcal{X}$. Now, we have to be cautious because the first term in the right hand side explodes as $\kappa_{1} \rightarrow \infty$. However, the trivial observation:

$\left|\mathbb{P}\left(Q_{p, 0}^{\kappa_{1}}\left(u_{n}\right) \cap\left(\cap_{j=2}^{\varsigma} \mathscr{N}_{u_{n}}\left(I_{j}\right)=\kappa_{j}\right)\right)-\mathbb{P}\left(Q_{p, 0}^{\kappa_{1}}\left(u_{n}\right)\right) \mathbb{P}\left(\cap_{j=2}^{\varsigma} \mathscr{N}_{u_{n}}\left(I_{j}\right)=\kappa_{j}\right)\right| \leq 2 \mathbb{P}\left(Q_{p, 0}^{\kappa_{1}}\left(u_{n}\right)\right)$, allows us to set:

$\gamma(n, t)=\min _{\kappa_{1} \in \mathbb{N}_{0}}\left\{2 \theta(1-\theta)^{\kappa_{1}} \mathbb{P}\left(X_{0}>u_{n}\right), C\left(\left(\frac{n}{(1-\theta)^{\kappa_{1}}}\right)^{\beta(1+\eta)} \varrho(t)+\left(\frac{(1-\theta)^{\kappa_{1}}}{n}\right)^{\Theta(d+\eta)}\right)\right\}$.

Since by assumption, there exists a sequence $\left(t_{n}\right)_{n \in \mathbb{N}}$ such that $n^{1+\beta(1+\eta)} \varrho\left(t_{n}\right)+n^{-\delta} \underset{n \rightarrow \infty}{\longrightarrow} 0$, then $n \gamma\left(n, t_{n}\right) \rightarrow 0$ as $n \rightarrow \infty$, as required.

5.2. Dichotomy for uniformly and piecewise expanding systems. In this section we prove Theorems 8 and 9 . We begin with the following notion:

Definition 6. For every $A \in \mathcal{B}$, we define the first return time to $A$, which we denote by $R(A)$, as the minimum of the return time function to $A$, i.e.,

$$
R(A)=\min _{x \in A} r_{A}(x) .
$$

Proposition 5.3. Consider a dynamical system $(\mathcal{X}, \mathcal{B}, \mathbb{P}, f)$ for which there exists decay of correlations against $L^{1}$ as in the assumptions of Theorems 8 and 9 . For any point $\zeta$, assume that (R1) holds and, in case $\zeta$ is periodic, that (R2) also holds. Consider that 
$X_{0}, X_{1}, \ldots$ is defined as in (3.1), let $u_{n}$ be such that (2.3) holds. For every $n \in \mathbb{N}$, if $\zeta$ is not periodic take $A_{n}=U_{n}:=U\left(u_{n}\right)$ and, if $\zeta$ is periodic of period $p$, let $A_{n}=Q_{p}\left(u_{n}\right)$. Also, set $R_{n}:=R\left(U_{n}\right)$, when $\zeta$ is non-periodic and $R_{n}:=\min _{x \in Q_{p}\left(u_{n}\right)} r_{U_{n}}(x)$, when $\zeta$ is periodic of prime period $p$.

If there exists $C^{\prime}>0$ such that for all $n$ we have $\mathbf{1}_{A_{n}} \in \mathcal{C},\left\|\mathbf{1}_{A_{n}}\right\|_{\mathcal{C}} \leq C^{\prime}$ and $R_{n} \rightarrow \infty$, as $n \rightarrow \infty$, then condition $D^{\prime}\left(u_{n}\right)$ holds for $X_{0}, X_{1}, \ldots$, when $\zeta$ is not periodic and $D_{p}^{\prime}\left(u_{n}\right)^{*}$ holds, when $\zeta$ is periodic of period $p$.

Proof. Taking $\phi=\mathbf{1}_{A_{n}}$ and $\psi=\mathbf{1}_{U_{n}}$ in (5.1) and since $\left\|\mathbf{1}_{A_{n}}\right\|_{\mathcal{C}} \leq C^{\prime}$ we easily get

$$
\mathbb{P}\left(A_{n} \cap f^{-j}\left(U_{n}\right)\right) \leq\left(\mathbb{P}\left(U_{n}\right)\right)^{2}+C\left\|\mathbf{1}_{A_{n}}\right\|_{\mathcal{C}}\left\|\mathbf{1}_{U_{n}}\right\|_{L^{1}(\mathbb{P})} j^{-2} \leq\left(\mathbb{P}\left(U_{n}\right)\right)^{2}+C^{*} \mathbb{P}\left(U_{n}\right) j^{-2},
$$

where $C^{*}=C C^{\prime}>0$. By definition of $R_{n}$, estimate (5.5) and since $n \mathbb{P}\left(U_{n}\right) \rightarrow \tau$ as $n \rightarrow \infty$ it follows that there exists some constant $c>0$ such that

$$
\begin{gathered}
n \sum_{j=1}^{\left\lfloor n / k_{n}\right\rfloor} \mathbb{P}\left(A_{n} \cap f^{-j}\left(U_{n}\right)\right)=n \sum_{j=R_{n}}^{\left\lfloor n / k_{n}\right\rfloor} \mathbb{P}\left(A_{n} \cap f^{-j}\left(U_{n}\right)\right) \leq n\left\lfloor\frac{n}{k_{n}}\right\rfloor \mathbb{P}\left(U_{n}\right)^{2}+n C^{*} \mathbb{P}\left(U_{n}\right) \sum_{j=R_{n}}^{\left\lfloor n / k_{n}\right\rfloor} j^{-2} \\
\leq \frac{\left(n \mathbb{P}\left(U_{n}\right)\right)^{2}}{k_{n}}+n C^{*} \mathbb{P}\left(U_{n}\right) \sum_{j=R_{n}}^{\infty} j^{-2} \leq c\left(\frac{\tau^{2}}{k_{n}}+\tau \sum_{j=R_{n}}^{\infty} j^{-2}\right) \underset{n \rightarrow \infty}{\longrightarrow} 0 .
\end{gathered}
$$

Lemma 5.4. Assume that $\zeta$ is not a periodic point and that $f$ is continuous at every point of the orbit of $\zeta$, namely $\zeta, f(\zeta), f^{2}(\zeta), \ldots$, then $\lim _{n \rightarrow \infty} R\left(U\left(u_{n}\right)\right)=\infty$.

Proof. Note that, by hypothesis, $f^{i}$ is continuous at $\zeta$, for all $i \in \mathbb{N}$. For some fixed $j$, we define $\epsilon=\min _{i=1, \ldots, j} \operatorname{dist}\left(f^{i}(\zeta), \zeta\right)$ Then, using the continuity of each $f^{i}$ at $\zeta$, for every $i=1, \ldots, j$, there exists $\delta_{i}>0$ such that $f^{i}\left(B_{\delta_{i}}(\zeta)\right) \subset B_{\epsilon / 2}\left(f^{i}(\zeta)\right)$. Now, let $U:=\cap_{i=1}^{j} B_{\delta_{i}}(\zeta)$

If we choose $N$ sufficiently large so that $U_{n} \subset U$ for all $n \geq N$ then, by definition of $\epsilon$ it is clear that $f^{i}\left(U_{n}\right) \cap U_{n}=\emptyset$, for all $i=1, \ldots, j$, which implies that $R\left(U\left(u_{n}\right)\right)>j$.

Proof of Theorems 8 and 9. As explained in Section 5.1, conditions $D_{2}\left(u_{n}\right), D_{3}\left(u_{n}\right), D^{p}\left(u_{n}\right)$, $D^{p}\left(u_{n}\right)^{*}$ are designed to follow easily from decay of correlations and we even do not need such strong version as decay against $L^{1}$. In any case, if we choose $\phi=\mathbf{1}_{A_{n}}\left(\phi=\mathbf{1}_{Q_{p}^{\kappa_{1}}\left(u_{n}\right)}\right.$, in the case of $\left.D^{p}\left(u_{n}\right)^{*}\right)$ and $\psi=\mathbf{1}_{\mathscr{W}_{0, \ell}\left(A_{n}\right)}\left(\psi=\mathbf{1}_{\mathscr{N}(A)=0}\right.$ in the case of $D_{3}\left(u_{n}\right)$ and

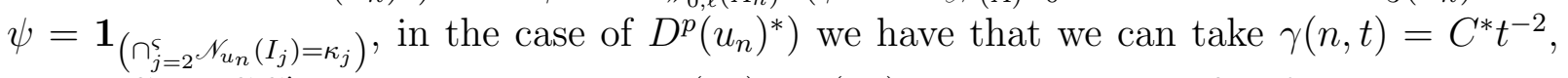
where $C^{*}=C C^{\prime}$. Hence, conditions $D_{2}\left(u_{n}\right), D^{p}\left(u_{n}\right)$ are trivially satisfied for the sequence $\left(t_{n}\right)_{n}$ given by $t_{n}=n^{2 / 3}$, for example.

To end the proof we only need to check the hypothesis of Proposition 5.3 . For that purpose, note that by Proposition 5.4 we have $R\left(U\left(u_{n}\right)\right) \underset{n \rightarrow \infty}{\longrightarrow} \infty$. 
Regarding the case when $\zeta$ is periodic point of prime period $p$, by the Hartman-Grobman theorem there is a neighbourhood $V$ around $\zeta$ where $f$ is conjugate to its linear approximation given by the derivative at $\zeta$. Hence, for $n$ sufficiently large so that $U\left(u_{n}\right) \subset V$, if a point starts in $Q_{p}\left(u_{n}\right)$ it takes a time $\alpha_{n}$ to leave $V$, during which, it is guaranteed that it does not return to $U\left(u_{n}\right)$. Moreover, since by condition (R1) and definition of $u_{n}$, we have that $U\left(u_{n}\right)$ shrinks to $\zeta$ as $n \rightarrow \infty$, then $\alpha_{n} \rightarrow \infty$ as $n \rightarrow \infty$. Since $R_{n} \geq \alpha_{n}$ then $R_{n} \underset{n \rightarrow \infty}{\longrightarrow} \infty$. This gives that $D_{p}^{\prime}\left(u_{n}\right)^{*}$ holds which implies that $D_{p}^{\prime}\left(u_{n}\right)$ holds as well.

5.3. Examples of specific dynamical systems. Let $f: \mathcal{X} \rightarrow \mathcal{X}$ be a measurable function as above. For a measurable potential $\phi: \mathcal{X} \rightarrow \mathbb{R}$, we define the pressure of $(\mathcal{X}, f, \phi)$ to be

$$
P(\phi):=\sup _{\mathbb{P} \in \mathcal{M}_{f}}\left\{h(\mathbb{P})+\int \phi d \mathbb{P}:-\int \phi d \mathbb{P}<\infty\right\},
$$

where $h(\mathbb{P})$ denotes the metric entropy of the measure $\mathbb{P}$, see [55] for details. If $\mathbb{P}$ is an invariant probability measure such that $h\left(\mathbb{P}_{\phi}\right)+\int \phi d \mathbb{P}=P(\phi)$, then we say that $\mathbb{P}$ is an equilibrium state for $(\mathcal{X}, f, \phi)$.

A measure $m$ is called a $\phi$-conformal measure if $m(\mathcal{X})=1$ and if whenever $f: A \rightarrow f(A)$ is a bijection, for a Borel set $A$, then $m(f(A))=\int_{A} e^{-\phi} d m$. Therefore, setting

$$
S_{n} \phi(x):=\phi(x)+\cdots+\phi \circ f^{n-1}(x),
$$

if $f^{n}: A \rightarrow f^{n}(A)$ is a bijection then $m\left(f^{n}(A)\right)=\int_{A} e^{-S_{n} \phi} d m$.

Note that for example for a smooth map interval map $f$, Lebesgue measure is $\phi$-conformal for $\phi(x):=-\log |D f(x)|$. Moreover, if for example $f$ is a topologically transitive quadratic interval map then as in Ledrappier [47], any acip $\mathbb{P}$ with $h(\mathbb{P})>0$ is an equilibrium state for $\phi$. This also holds for the even simpler case of piecewise smooth uniformly expanding maps, which we consider below. This is the case we principally consider in this paper. For results on more general equilibrium states see [28].

5.3.1. Rychlik systems. The first class of examples to which we apply our results is the class of interval maps considered by Rychlik in [51, that is given by a triple $(Y, f, \phi)$, where $Y$ is an interval, $f$ a piecewise expanding interval map (possibly with countable discontinuity points) and $\phi$ a certain potential. This class includes, for example, piecewise $C^{2}$ uniformly expanding maps of the unit interval with the relevant physical measures. We refer to [51] or to [30, Section 4.1] for details on the definition of such class and instead give the following list of examples of maps in such class:

- Given $m \in\{2,3, \ldots\}$, let $f: x \mapsto m x \bmod 1$ and $\phi \equiv-\log m$. Then $m_{\phi}=\mathbb{P}_{\phi}=$ Leb.

- Let $f: x \mapsto 2 x \bmod 1$ and for $\alpha \in(0,1)$, let

$$
\phi(x):= \begin{cases}-\log \alpha & \text { if } x \in(0,1 / 2) \\ -\log (1-\alpha) & \text { if } x \in(1 / 2,1)\end{cases}
$$

(and $\phi=-\infty$ elsewhere). Then $m_{\phi}=\mathbb{P}_{\phi}$ is the $(\alpha, 1-\alpha)$-Bernoulli measure on $[0,1]$. 
- Let $f:(0,1] \rightarrow(0,1]$ and $\phi:(-\infty, 0)$ be defined as $f(x)=2^{k}\left(x-2^{-k}\right)$ and $\phi(x):=$ $-k \log 2$ for $x \in\left(2^{-k}, 2^{-k+1}\right]$. Then $m_{\phi}=\mathbb{P}_{\phi}=$ Leb.

In order to prove that the stated dichotomies hold for these systems, we basically need to show that these systems satisfy the conditions Theorems 8 and 9 .

In this setting, as in [51], there is a unique $f$-invariant probability measure $\mathbb{P}_{\phi} \ll m_{\phi}$ which is also an equilibrium state for $(Y, f, \phi)$ with a strictly positive density $\frac{d \mathbb{P}_{\phi}}{d m_{\phi}} \in B V$. Moreover, there exists exponential decay of correlations against $L^{1}\left(m_{\phi}\right)$, i.e., there exist $C>0$ and $\beta>0$, such that for any $v \in B V$ and $\psi \in L^{1}\left(m_{\phi}\right)$ we have

$$
\left|\int \psi \circ f^{n} \cdot v d \mathbb{P}_{\phi}-\int \psi d \mathbb{P}_{\phi} \int v d \mathbb{P}_{\phi}\right| \leq C\|v\|_{B V}\|\psi\|_{L^{1}\left(m_{\phi}\right)} \mathrm{e}^{-\beta n} .
$$

Assume that $\zeta$ is such that $0<\frac{d \mathbb{P}_{\phi}}{d m_{\phi}}(\zeta)<\infty$ and the observable $\varphi: \mathcal{X} \rightarrow \mathbb{R} \cup\{+\infty\}$ is of the form (4.2). The the regularity of $\mathbb{P}_{\phi}$ and $\varphi$ guarantee that condition (R1) holds for every such $\zeta$. Besides, if $\zeta$ is a repelling p-periodic point, which means that $f^{p}(\zeta)=\zeta, f^{p}$ is differentiable at $\zeta$ and $0<\left|\operatorname{det} D\left(f^{-p}\right)(\zeta)\right|<1$. As shown in [30, Theorem 5], we have that (R2) holds. Moreover, the EI is given by the formula $\theta=1-\mathrm{e}^{S_{p} \phi(\zeta)}$.

Finally, since $U\left(u_{n}\right)$ is an interval and $Q_{p, 0}^{\kappa_{1}}\left(u_{n}\right)$ is the union of two intervals, for all $\kappa_{1}$, we have that $\left\|\mathbf{1}_{U\left(u_{n}\right)}\right\|_{B V} \leq 3$ and $\left\|\mathbf{1}_{Q_{p, 0}^{\kappa_{1}\left(u_{n}\right)}}\right\|_{B V} \leq 5$, which means all the assumptions of Theorems 8 and 9 hold.

5.3.2. Piecewise expanding maps in higher dimensions. The second class of examples we consider here corresponds to a higher dimensional version of the piecewise expanding interval maps of the previous section. We refer to [52, Section 2] for precise definition of this class of maps and give a very particular example corresponding to a uniformly expanding map on the 2-dimensional torus:

- let $\mathbb{T}^{2}=\mathbb{R}^{2} / \mathbb{Z}^{2}$ and consider the map $f: \mathbb{T}^{2} \rightarrow \mathbb{T}^{2}$ defined by the action of a $2 \times 2$ matrix with integer entries and eigenvalues $\lambda_{1}, \lambda_{2}>1$.

According to [52, Theorem 5.1], there exists an acip $\mathbb{P}$. Also in [52, Theorem 6.1], it is shown that on the mixing components, $\mathbb{P}$ enjoys exponential decay of correlations against $L^{1}$ observables on $V_{\alpha}$, more precisely, if the map $f$ is as defined above and if $\mathbb{P}$ is the mixing acip, then there exist constants $C<\infty$ and $\gamma<1$ such that

$$
\left|\int \psi \circ f^{n} h \mathrm{~d} \mathbb{P}\right| \leq C\|\psi\|_{L^{1}}\|h\|_{\alpha} \gamma^{n}, \forall \psi \in L^{1}, \text { where } \int \psi \mathrm{d} \mathbb{P}=0 \text { and } \forall h \in V_{\alpha} .
$$

Assume that the observable $\varphi: \mathcal{X} \rightarrow \mathbb{R} \cup\{+\infty\}$ is of the form 4.2). This guarantees that condition (R1) holds. If $\zeta$ is a repelling p-periodic point, which means that $f^{p}(\zeta)=\zeta, f^{p}$ is differentiable at $\zeta$ and $0<\left|\operatorname{det} D\left(f^{-p}\right)(\zeta)\right|<1$. Then condition (R2) holds and the EI is equal to $\theta=1-\left|\operatorname{det} D\left(f^{-p}\right)(\zeta)\right|$ (see [30, Theorem 3]). It is also easy to check that $\left\|\mathbf{1}_{U\left(u_{n}\right)}\right\|_{\alpha}$ and $\left\|\mathbf{1}_{Q_{p, 0}^{\kappa_{1}}\left(u_{n}\right)}\right\|_{\alpha}$ are bounded by a positive constant, for all $\kappa_{1}$, which means that all conditions of Theorems 8 and 9 hold. 


\section{REFERENCES}

[1] M. Abadi. Sharp error terms and necessary conditions for exponential hitting times in mixing processes. Ann. Probab., 32(1A):243-264, 2004.

[2] M. Abadi. Hitting, returning and the short correlation function. Bull. Braz. Math. Soc. (N.S.), 37(4):593-609, 2006.

[3] M. Abadi and A. Galves. Inequalities for the occurrence times of rare events in mixing processes. The state of the art. Markov Process. Related Fields, 7(1):97-112, 2001. Inhomogeneous random systems (Cergy-Pontoise, 2000).

[4] M. Abadi and B. Saussol. Hitting and returning to rare events for all alpha-mixing processes. Stochastic Process. Appl., 121(2):314-323, 2011.

[5] M. Abadi and S. Vaienti. Large deviations for short recurrence. Discrete Contin. Dyn. Syst., 21(3):729$747,2008$.

[6] M. Abadi and N. Vergne. Sharp error terms for return time statistics under mixing conditions. $J$. Theoret. Probab., 22(1):18-37, 2009.

[7] J. F. Alves, J. M. Freitas, S. Luzzatto, and S. Vaienti. From rates of mixing to recurrence times via large deviations. Adv. Math., 228(2):1203-1236, 2011.

[8] H. Aytaç, J. M. Freitas, and S. Vaienti. Laws of rare events for deterministic and random dynamical systems. Preprint arXiv:1207.5188, 2012.

[9] R. C. Bradley. Basic properties of strong mixing conditions. A survey and some open questions. Probab. Surv., 2:107-144 (electronic), 2005. Update of, and a supplement to, the 1986 original.

[10] H. Bruin, B. Saussol, S. Troubetzkoy, and S. Vaienti. Return time statistics via inducing. Ergodic Theory Dynam. Systems, 23(4):991-1013, 2003.

[11] H. Bruin and M. Todd. Return time statistics of invariant measures for interval maps with positive Lyapunov exponent. Stoch. Dyn., 9(1):81-100, 2009.

[12] H. Bruin and S. Vaienti. Return time statistics for unimodal maps. Fund. Math., 176(1):77-94, 2003.

[13] J.-R. Chazottes and P. Collet. Poisson approximation for the number of visits to balls in non-uniformly hyperbolic dynamical systems. Ergodic Theory Dynam. Systems, FirstView, 2012.

[14] Z. Coelho. Asymptotic laws for symbolic dynamical systems. In Topics in symbolic dynamics and applications (Temuco, 1997), volume 279 of London Math. Soc. Lecture Note Ser., pages 123-165. Cambridge Univ. Press, Cambridge, 2000.

[15] P. Collet. Some ergodic properties of maps of the interval. In Dynamical systems (Temuco, 1991/1992), volume 52 of Travaux en Cours, pages 55-91. Hermann, Paris, 1996.

[16] P. Collet. Statistics of closest return for some non-uniformly hyperbolic systems. Ergodic Theory Dynam. Systems, 21(2):401-420, 2001.

[17] M. Denker, M. Gordin, and A. Sharova. A Poisson limit theorem for toral automorphisms. Illinois J. Math., 48(1):1-20, 2004.

[18] D. Dolgopyat. Limit theorems for partially hyperbolic systems. Trans. Amer. Math. Soc., 356(4):16371689 (electronic), 2004.

[19] D. Faranda, J. M. Freitas, V. Lucarini, G. Turchetti, and S. Vaienti. Extreme value statistics for dynamical systems with noise. Preprint arXiv:1208.5582, 2012.

[20] D. Faranda, V. Lucarini, G. Turchetti, and S. Vaienti. Generalized extreme value distribution parameters as dynamical indicators of stability. To appear in Int. J. Bif. Chaos. (arXiv: 1107.5972v1), 2011.

[21] D. Faranda, V. Lucarini, G. Turchetti, and S. Vaienti. Numerical convergence of the block-maxima approach to the generalized extreme value distribution. J. Stat. Phys., 145:1156-1180, 2011.

[22] D. Faranda, V. Lucarini, G. Turchetti, and S. Vaienti. Extreme value distribution for singular measures. Chaos, 22(2):023135, 2012.

[23] A. Ferguson and M. Pollicott. Escape rates for Gibbs measures. Ergodic Theory Dynam. Systems, 32(3):961-988, 2012. 
[24] A. Fisher and L. Tippett. Limiting forms of the frequency distribution of the largest or smallest member of a sample. Math. Proc. Cambridge Philos. Soc., 24(2):180-190, 1928.

[25] A. C. M. Freitas and J. M. Freitas. Extreme values for Benedicks-Carleson quadratic maps. Ergodic Theory Dynam. Systems, 28(4):1117-1133, 2008.

[26] A. C. M. Freitas and J. M. Freitas. On the link between dependence and independence in extreme value theory for dynamical systems. Statist. Probab. Lett., 78(9):1088-1093, 2008.

[27] A. C. M. Freitas, J. M. Freitas, and M. Todd. Hitting time statistics and extreme value theory. Probab. Theory Related Fields, 147(3):675-710, 072010.

[28] A. C. M. Freitas, J. M. Freitas, and M. Todd. Extreme value laws in dynamical systems for non-smooth observations. J. Stat. Phys., 142(1):108-126, 2011.

[29] A. C. M. Freitas, J. M. Freitas, and M. Todd. The compound Poisson limit ruling periodic extreme behaviour of non-uniformly hyperbolic dynamics. To appear in Comm. Math. Phys. (arXiv:1204.2304), 2012.

[30] A. C. M. Freitas, J. M. Freitas, and M. Todd. The extremal index, hitting time statistics and periodicity. Adv. Math., 231(5):2626 - 2665, 2012.

[31] A. Galves and B. Schmitt. Occurrence times of rare events for mixing dynamical systems. Ann. Inst. H. Poincaré Phys. Théor., 52(3):267-281, 1990.

[32] B. Gnedenko. Sur la distribution limite du terme maximum d'une série aléatoire. Ann. of Math. (2), 44:423-453, 1943.

[33] C. Gupta, M. Holland, and M. Nicol. Extreme value theory and return time statistics for dispersing billiard maps and flows, Lozi maps and Lorenz-like maps. Ergodic Theory Dynam. Systems, 31(5):13631390, 2011.

[34] N. Haydn, Y. Lacroix, and S. Vaienti. Hitting and return times in ergodic dynamical systems. Ann. Probab., 33(5):2043-2050, 2005.

[35] N. Haydn and S. Vaienti. The compound Poisson distribution and return times in dynamical systems. Probab. Theory Related Fields, 144(3-4):517-542, 2009.

[36] M. Hirata. Poisson law for Axiom A diffeomorphisms. Ergodic Theory Dynam. Systems, 13(3):533-556, 1993.

[37] M. Hirata, B. Saussol, and S. Vaienti. Statistics of return times: a general framework and new applications. Comm. Math. Phys., 206(1):33-55, 1999.

[38] M. Holland, M. Nicol, and A. Török. Extreme value theory for non-uniformly expanding dynamical systems. Trans. Amer. Math. Soc., 364:661-688, 2012.

[39] M. P. Holland, R. Vitolo, P. Rabassa, A. E. Sterk, and H. W. Broer. Extreme value laws in dynamical systems under physical observables. Physica D: Nonlinear Phenomena, 241(5):497 - 513, 2012.

[40] O. Kallenberg. Random measures. Akademie-Verlag, Berlin, fourth edition, 1986.

[41] G. Keller. Rare events, exponential hitting times and extremal indices via spectral perturbationâ. Dynamical Systems, 27(1):11-27, 2012.

[42] G. Keller and C. Liverani. Rare events, escape rates and quasistationarity: some exact formulae. $J$. Stat. Phys., 135(3):519-534, 2009.

[43] Y. Kifer and A. Rapaport. Poisson and compound Poisson approximations in a nonconventional setup. Preprint arXiv:1211.5238, November 2012.

[44] M. R. Leadbetter. On extreme values in stationary sequences. Z. Wahrscheinlichkeitstheorie und Verw. Gebiete, 28:289-303, 1973/74.

[45] M. R. Leadbetter. Extremes and local dependence in stationary sequences. Z. Wahrsch. Verw. Gebiete, 65(2):291-306, 1983.

[46] M. R. Leadbetter, G. Lindgren, and H. Rootzén. Extremes and related properties of random sequences and processes. Springer Series in Statistics. Springer-Verlag, New York, 1983.

[47] F. Ledrappier. Some properties of absolutely continuous invariant measures on an interval. Ergodic Theory Dynamical Systems, 1(1):77-93, 1981. 
[48] R. M. Loynes. Extreme values in uniformly mixing stationary stochastic processes. Ann. Math. Statist., 36:993-999, 1965.

[49] V. Lucarini, D. Faranda, and J. Wouters. Universal behavior of extreme value statistics for selected observables of dynamical systems. J. Stat. Phys., 147(1):63-73, 2012.

[50] B. Pitskel'. Poisson limit law for Markov chains. Ergodic Theory Dynam. Systems, 11(3):501-513, 1991.

[51] M. Rychlik. Bounded variation and invariant measures. Studia Math., 76(1):69-80, 1983.

[52] B. Saussol. Absolutely continuous invariant measures for multidimensional expanding maps. Israel J. Math., 116:223-248, 2000.

[53] B. Saussol. An introduction to quantitative Poincaré recurrence in dynamical systems. Rev. Math. Phys., 21(8):949-979, 2009.

[54] R. Vitolo, M. P. Holland, and C. A. T. Ferro. Robust extremes in chaotic deterministic systems. Chaos, 19(4):043127, 2009.

[55] P. Walters. An introduction to ergodic theory, volume 79 of Graduate Texts in Mathematics. SpringerVerlag, New York, 1982.

Jorge Milhazes Freitas, Centro de Matemática \& Faculdade de Ciências da Universidade do Porto, Rua do Campo Alegre 687, 4169-007 Porto, Portugal

E-mail address: jmfreita@fc.up.pt

$U R L:$ http://www.fc.up.pt/pessoas/jmfreita 